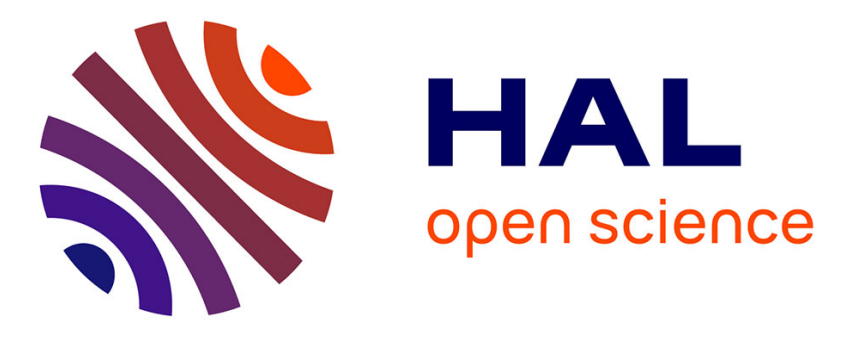

\title{
Unified semi-analytical wall boundary conditions for inviscid, laminar or turbulent flows in the meshless SPH method
}

\author{
Martin Ferrand, Dominique R. Laurence, Benedict D. Rogers, Damien \\ Violeau, Christophe Kassiotis
}

\section{To cite this version:}

Martin Ferrand, Dominique R. Laurence, Benedict D. Rogers, Damien Violeau, Christophe Kassiotis. Unified semi-analytical wall boundary conditions for inviscid, laminar or turbulent flows in the meshless SPH method. International Journal for Numerical Methods in Fluids, 2013, 71 (476-472), pp. Online. 10.1002/fld.3666 . hal-00691603

\section{HAL Id: hal-00691603 \\ https://hal-enpc.archives-ouvertes.fr/hal-00691603}

Submitted on 26 Apr 2012

HAL is a multi-disciplinary open access archive for the deposit and dissemination of scientific research documents, whether they are published or not. The documents may come from teaching and research institutions in France or abroad, or from public or private research centers.
L'archive ouverte pluridisciplinaire HAL, est destinée au dépôt et à la diffusion de documents scientifiques de niveau recherche, publiés ou non, émanant des établissements d'enseignement et de recherche français ou étrangers, des laboratoires publics ou privés. 


\title{
Unified semi-analytical wall boundary conditions for inviscid, laminar or turbulent flows in the meshless SPH method.
}

\author{
M. Ferrand, D. R. Laurence, B. D. Rogers, D. Violeau, C. Kassiotis, \\ E-mail: martin.ferrand@edf.fr
}

May 24, 2011

\section{Introduction}

Treating wall boundary conditions is one of the most challenging parts of the Smoothed Particle Hydrodynamics ( $S P H)$ method and many different approaches have been recently developed (see e.g. $[1,2,3,4,5,6]$ ). Accurate boundary conditions are essential since in many applications where precise loading on walls is required such as forces on floating bodies or shoreline structures, tank walls, wind-wave exchanges, fluid-structure interactions in power-plants etc. It is an obvious prerequisite to improve turbulence modelling near walls.

Many methods for implementing solid walls in $S P H$ have been developed over the past two decades. Among the most popular and commonly used include three broad categories:

1. Repulsive forces such as Lennard-Jones potential [7],

2. Fictitious particles (or alternatively ghost particles) which fill the empty area of the kernel support behind a boundary with artificial particles with prescribed physical quantities (such as pressure and velocity) to enforce no slip or free slip condition,

3. Semi-analytical boundary conditions based on a variational formulation introduced by Kulasegaram et al. [1] where a wall renormalization of the equations is made with respect to the missing area of the kernel support. We will describe a variant of this method in $\S 3$, where intrinsic gradient and divergence operators are employed that ensure conservation properties.

All of these methods have advantages and drawbacks. The Lennard-Jones potential force originally describes the interaction between pairs of atoms and is used to model the repulsion between a fluid particle and a boundary particle. This method is easy to implement, even for complex geometries and computationally cheap. However, it leads to spurious behaviour: for instance it is 
impossible to maintain particles fixed along a vertical wall in the presence of gravity.

The fictitious particles recommended in $[8]$ prevents such a non-physical behaviour. However, the positioning of ghost particles in complex geometries can be particularly unwieldy, particularly in $3 D$. Moreover, the computational effort required is not negligible, given that we increase the number of particles to take into account in the discrete summations (1) and (3).

Finally, the semi-analytical approach is attractive thanks to its variational derivation which means that some physical quantities such as momentum will be automatically conserved. Unfortunately, the original attempt did not present a clear and simple way to compute renormalization terms introduced. Furthermore, the formulation proposed was not able to reproduce hydrostatic pressure fields or to take into account the shear stress along a wall.

This paper therefore takes the semi-analtycial approach of [1] and extends it so that the accuracy of the physical field such as the pressure next to walls is considerably improved, and the consistent manner developed for wall-correct operators allows us to perform simulations with turbulence models. This work will present three key advances:

- The time integration scheme used for the continuity equation requires particular attention, and as already mentioned by Vila [9], we prove there is no point in using a dependence in time of the particles' density if no kernel gradient corrections are added. Thus, by using a near-boundary kernelcorrected version of the time integration scheme of the form proposed in [9], long-time simulations ideally suited for turbulent flows in the context of accurate boundary conditions are possible.

- To compute the kernel correction, Feldman and Bonet [10] use an analytical value which is computationally expensive whereas Kulasegaram et al. [1] and De Leffe et al. [6] use polynomial approximation which can be difficult to define for complex geometries. We propose here to compute the renormalisation term of the kernel support near a solid with a novel time integration scheme, allowing us any shape for the boundary.

- All boundary terms issued from the continuous approximation are given by surface summations which only require information from a $C A D$ mesh file of the boundary. The technique developed here allows us to correct the pressure gradient and viscous terms and hence provide a physically correct wall-shear stress so that even the diffusion equation of a scalar quantity can be solved accurately using $S P H$ such as the turbulent kinetic energy or its dissipation in a $k-\epsilon$ model of turbulence.

This paper is organised as follows: In the next Section we introduce the formulations for weakly compressible $S P H$ introducing the basic discretisations for operators, viscous forces, and turbulence modelling. In the Section following, we then develop the consistent boundary conditions with improved time integration and a position-dependent technique to compute the density. The paper then presents the computation of the renormalization terms using a novel time integration scheme before presenting the enhanced behaviour in the Section on numerical results. 


\section{Basic $S P H$ formulations for weakly compress- ible Newtonian fluid}

\subsection{Conservative governing equations}

The slightly compressible Newtonian fluid is modelled by a set of particles denoted by the subscripts $(.)_{a}$ and $(.)_{b}$ in a domain $\Omega$. The set of all the fluid particles is denoted by $\mathcal{F}$ where each particle $a \in \mathcal{F}$ possesses information such as its mass $m_{a}$ (assumed constant), its position $\mathbf{r}_{a}$, its velocity $\mathbf{u}_{a}$ (the Lagrangian derivative of the position), its density $\rho_{a}$, its volume $V_{a}=\frac{m_{a}}{\rho_{a}}$, and its pressure $p_{a}$. The spatial discretization is based on a weighting interpolation or kernel function, $w$, with compact support. $\Omega_{a}$ then refers to the support of the kernel function centred or $\mathbf{r}_{a}$ of radius $R$. We generally denote by the subscripts (.) $)_{a b}$ the difference of a quantity between the positions $a$ and $b$. For instance $\mathbf{u}_{a b} \equiv \mathbf{u}_{a}-\mathbf{u}_{b}$ and $\mathbf{r}_{a b} \equiv \mathbf{r}_{a}-\mathbf{r}_{b}$. Two exceptions are made with the following notations $w_{a b} \equiv w\left(\mathbf{r}_{a b}\right)$ and $\boldsymbol{\nabla} w_{a b} \equiv \nabla_{a} w\left(\mathbf{r}_{a b}\right)$. Here, the symbol $\boldsymbol{\nabla}_{a}$ denotes the gradient at the point $\mathbf{r}_{a}{ }^{1}$. With these notations, a commonly used form of the continuity equation is (see e.g. [11]):

$$
\frac{\mathrm{d} \rho_{a}}{\mathrm{~d} t}=\sum_{b \in \mathcal{F}} m_{b} \nabla w_{a b} \cdot \mathbf{u}_{a b}
$$

where $\frac{\mathrm{d}}{\mathrm{d} t}$ denotes the Lagrangian derivative, that is to say the derivative along the particle path. It can be derived from the following definition of the density:

$$
\rho_{a}=\sum_{b \in \mathcal{F}} m_{b} w_{a b}
$$

The inviscid momentum equation can be written as follows:

$$
\frac{\mathrm{d} \mathbf{u}_{a}}{\mathrm{~d} t}=-\sum_{b \in \mathcal{F}} m_{b}\left(\frac{p_{a}}{\rho_{a}^{2}}+\frac{p_{b}}{\rho_{b}^{2}}\right) \nabla w_{a b}+\mathbf{g}
$$

where $\mathbf{g}$ is gravity. The equation of state links density and pressure:

$$
p_{a}=\frac{\rho_{0} c_{0}^{2}}{\gamma}\left[\left(\frac{\rho_{a}}{\rho_{0}}\right)^{\gamma}-1\right]
$$

where $\rho_{0}$ is the reference density of the fluid, $c_{0}$ is the speed of sound and $\gamma=7$ is generally chosen for water.

\subsection{Viscous forces}

The viscous term is used in this work in $S P H$ for computing the viscous term $\frac{1}{\rho} \nabla \cdot(\mu \nabla \mathbf{u})$ is due to Morris et al. (see [12]):

$$
\frac{1}{\rho_{a}} \nabla \cdot(\mu \nabla \mathbf{u})_{a}=\sum_{b \in \mathcal{F}} m_{b} \frac{\mu_{a}+\mu_{b}}{\rho_{a} \rho_{b}} \frac{\mathbf{u}_{a b}}{r_{a b}^{2}} \mathbf{r}_{a b} \cdot \nabla w_{a b}
$$

\footnotetext{
${ }^{1}$ the gradient operator is defined by $\nabla_{a} \equiv \mathbf{e}_{x} \frac{\partial}{\partial x_{a}}+\mathbf{e}_{y} \frac{\partial}{\partial y_{a}}+\mathbf{e}_{z} \frac{\partial}{\partial z_{a}},\left(\mathbf{e}_{x}, \mathbf{e}_{y}, \mathbf{e}_{z}\right)$ being the basis vector triad of the Cartesian coordinate system in $3 D$.
} 
where the dynamic viscosity $\mu$ is given by:

$$
\mu \equiv \nu \rho
$$

and $\nu$ is the kinematic molecular viscosity.

Operator definitions: For later analysis, we define discrete operators gradient $\left(\operatorname{Grad}_{a}\left\{A_{b}\right\}\right)$, divergence $\left(\operatorname{Div}_{a}\left\{\mathbf{A}_{b}\right\}\right)$ and Laplacian $\left(\operatorname{Lap}_{a}\left(\left\{B_{b}\right\},\left\{\mathbf{A}_{b}\right\}\right)\right)$ of arbitrary discrete scalar $\left(\left\{A_{b}\right\}\right.$ and $\left.\left\{B_{b}\right\}\right)$ or vector fields $\left(\left\{\mathbf{A}_{b}\right\}\right)$ as:

$$
\begin{aligned}
\operatorname{Grad}_{a}\left\{A_{b}\right\} & \equiv \rho_{a} \sum_{b \in \mathcal{F}} m_{b}\left(\frac{A_{a}}{\rho_{a}^{2}}+\frac{A_{b}}{\rho_{b}^{2}}\right) \nabla w_{a b} \\
\operatorname{Div}_{a}\left\{\mathbf{A}_{b}\right\} & \equiv-\frac{1}{\rho_{a}} \sum_{b \in \mathcal{F}} m_{b} \mathbf{A}_{a b} \cdot \nabla w_{a b} \\
\operatorname{Lap}_{a}\left(\left\{B_{b}\right\},\left\{\mathbf{A}_{b}\right\}\right) & \equiv \rho_{a} \sum_{b \in \mathcal{F}} m_{b} \frac{B_{a}+B_{b}}{\rho_{a} \rho_{b}} \frac{\mathbf{A}_{a b}}{r_{a b}^{2}} \mathbf{r}_{a b} \cdot \nabla w_{a b}
\end{aligned}
$$

where $\mathbf{A}_{a b} \equiv \mathbf{A}_{a}-\mathbf{A}_{b}$. They are approximations of the continuous gradient, divergence and Laplacian operators (respectively denoted by $\boldsymbol{\nabla}_{a}, \boldsymbol{\nabla}$. and $\boldsymbol{\nabla} \cdot \boldsymbol{\nabla}$ ). Thus the equation of continuity (1) and the momentum equation (3) can be rewritten as follows:

$$
\begin{aligned}
\frac{\mathrm{d} \rho_{a}}{\mathrm{~d} t} & =-\rho_{a} \operatorname{Div}_{a}\left\{\mathbf{u}_{b}\right\} \\
\frac{\mathrm{d} \mathbf{u}_{a}}{\mathrm{~d} t} & =-\frac{1}{\rho_{a}} \operatorname{Grad}_{a}\left\{p_{b}\right\}+\mathbf{g}+\frac{1}{\rho_{a}} \operatorname{Lap}_{a}\left(\left\{\mu_{b}\right\},\left\{\mathbf{u}_{b}\right\}\right)
\end{aligned}
$$

The operators $\operatorname{Grad}_{a}$ and $\operatorname{Div}_{a}$ are said to be skew-adjoint. It is possible to define variants of these three operators conserving this property of adjunction (see [13]).

\subsection{Turbulence modelling in $S P H$}

The Reynolds approach consists in considering only the mean part ${ }^{2}$ denoted by $\overline{\mathbf{u}}$ of the velocity field $\mathbf{u}$ in the equation of Navier Stokes, then modelling the effects of the fluctuating part of the velocity field on the mean velocity.

The $k-\epsilon$ turbulence model developed by Launder et al. [14] add two transport equations of $k$, the turbulent kinetic energy, and $\epsilon$, its dissipation, to the momemtum equation (8) which is modified as follows:

$$
\begin{aligned}
\frac{\mathrm{D} \rho_{a}}{\mathrm{D} t} & =-\rho_{a} \operatorname{Div}_{a}\left\{\overline{\mathbf{u}}_{b}\right\} \\
\frac{\mathrm{D} \overline{\mathbf{u}}_{a}}{\mathrm{D} t} & =\frac{1}{\rho_{a}} \operatorname{Grad}_{a}\left\{\bar{p}_{b}+\frac{2}{3} \rho k_{b}\right\}+\operatorname{Lap}_{a}\left(\left\{\mu_{b}+\mu_{T b}\right\},\left\{\overline{\mathbf{u}}_{b}\right\}\right)+\mathbf{g} \\
\frac{\mathrm{D} k_{a}}{\mathrm{D} t} & =\frac{1}{\rho_{a}} \operatorname{Lap}_{a}\left(\left\{\mu_{b}+\frac{\mu_{T b}}{\sigma_{k}}\right\},\left\{k_{b}\right\}\right)+P_{a}-\epsilon_{a} \\
\frac{\mathrm{D} \epsilon_{a}}{\mathrm{D} t} & =\frac{1}{\rho_{a}} \operatorname{Lap}_{a}\left(\left\{\mu_{b}+\frac{\mu_{T b}}{\sigma_{\epsilon}}\right\},\left\{\epsilon_{b}\right\}\right)+\frac{\epsilon_{a}}{k_{a}}\left(C_{\epsilon 1} P_{a}-C_{\epsilon 2} \epsilon_{a}\right)
\end{aligned}
$$

\footnotetext{
${ }^{2}$ in the stochastic point of view.
} 
where the derivative $\frac{\mathrm{D}}{\mathrm{D} t} \equiv \frac{\partial}{\partial t}+\overline{\mathbf{u}} . \boldsymbol{\nabla}$ is the Lagrangian derivative along the Reynolds averaged field $\left\{\overline{\mathbf{u}}_{b}\right\}$. The $k-\epsilon$ model links the turbulent kinetic viscosity $\nu_{T} \equiv \frac{\mu_{T}}{\rho}$ to the turbulent kinetic energy $k$ and its dissipation $\epsilon$ by:

$$
\nu_{T a}=C_{\mu} \frac{k_{a}^{2}}{\epsilon_{a}}
$$

with constants $\sigma_{k}, \sigma_{\epsilon}, C_{\epsilon 1}$ and $C_{\epsilon 2}$ given by [14].

The production term of $k, P_{a}$, is defined by:

$$
P_{a}=\nu_{T a} S_{a}^{2}
$$

where $S_{a}^{2} \equiv 2 \mathbf{S}_{a}: \mathbf{S}_{a}$ is the scalar mean rate-of-strain. The tensorial strain rate of the mean velocity field is given by $\mathbf{S}_{a} \equiv \frac{1}{2}\left(\boldsymbol{\nabla}_{a} \overline{\mathbf{u}}+\boldsymbol{\nabla}_{a} \overline{\mathbf{u}}^{T}\right)$; Violeau and Issa [8] discretized in the $S P H$ form the velocity gradient:

$$
\operatorname{Grad}_{a}\left\{\overline{\mathbf{u}}_{b}\right\} \equiv-\frac{1}{\rho_{a}} \sum_{b \in \mathcal{F}} m_{b} \overline{\mathbf{u}}_{a b} \otimes \nabla w_{a b}
$$

In the following, for sake of simplicity we will drop all overbars to denote velocity and pressure, and thus denote Lagrangian derivatives $\frac{\mathrm{d}}{\mathrm{d} t}$. The reader should keep in mind that under turbulent conditions, the latter quantities are considered as Reynolds-averaged and the Lagrangian derivative is along the Reynolds averaged velocity field.

\section{New boundary conditions and accurate time stepping}

\subsection{Derivation of wall boundary terms using continuous interpolation}

\subsubsection{Kulasegaram et al.'s renormalisation:}

Instead of assuming that $\rho_{a} \simeq \sum_{b \in \mathcal{F}} m_{b} w_{a b}$, which underestimates $\rho_{a}$ when the particle $a$ is close to a boundary (see Figure 1), Kulasegaram et al. [1] renormalise the estimation using a function $\gamma_{a}$ :

$$
\rho_{a} \simeq \frac{1}{\gamma_{a}} \sum_{b \in \mathcal{F}} m_{b} w_{a b}
$$

where $\gamma_{a}$ is defined by:

$$
\gamma_{a} \equiv \int_{\Omega \cap \Omega_{a}} w\left(\mathbf{r}^{\prime}-\mathbf{r}_{a}\right) \mathrm{d} V^{\prime}
$$

In general, $\gamma_{a}$ is an Eulerian field depending only on the position of the particle $a$ with respect to boundaries of $\Omega$. Far from a solid boundary $\gamma_{a}=1$. 


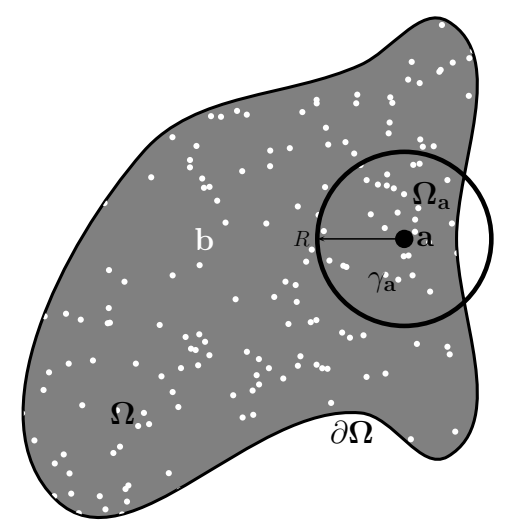

Figure 1: Kernel-boundary interaction.

The introduction of $\gamma_{a}$ into the derivation of the governing equations leads to some key differences such as the new continuity equation [1] where starting from (13) is rewritten:

$$
\frac{\mathrm{d} \rho_{a}}{\mathrm{~d} t}=\frac{1}{\gamma_{a}} \sum_{b \in \mathcal{F}} m_{b} \boldsymbol{\nabla} w_{a b} \cdot \mathbf{u}_{a b}-\frac{\rho_{a}}{\gamma_{a}} \nabla \gamma_{a} \cdot \mathbf{u}_{a}
$$

This can be compared with (1). Accordingly, the gradient, $\nabla \gamma_{a}$ is defined by:

$$
\nabla \gamma_{a} \equiv \int_{\Omega \cap \Omega_{a}} \nabla_{a} w\left(\mathbf{r}^{\prime}-\mathbf{r}_{a}\right) \mathrm{d} V^{\prime}=\int_{\partial \Omega \cap \Omega_{a}} w\left(\mathbf{r}^{\prime}-\mathbf{r}_{a}\right) \mathbf{n d} S^{\prime}
$$

where the second integral is obtained using the Gauss theorem and where $\mathbf{n}$ is the inward boundary normal.

In order to evaluate the new internal forces and contact forces, Kulasegaram et al. [1] derive the internal energy using the equation of Lagrange. They obtain a new internal force due to the pressure. The correction of Kulasegaram et al. can be expressed in terms of new compatible (in a variational sense) operators gradient and divergence. Indeed, one can define:

$$
\begin{aligned}
\operatorname{Grad}_{a}^{K}\left\{A_{b}\right\} & \equiv \rho_{a} \sum_{b \in \mathcal{F}} m_{b}\left(\frac{A_{a}}{\gamma_{a} \rho_{a}^{2}}+\frac{A_{b}}{\gamma_{b} \rho_{b}^{2}}\right) \nabla w_{a b}-\frac{A_{a}}{\gamma_{a}} \nabla \gamma_{a} \\
\operatorname{Div}_{a}^{K}\left\{\mathbf{A}_{b}\right\} & \equiv-\frac{1}{\gamma_{a} \rho_{a}} \sum_{b \in \mathcal{F}} m_{b} \mathbf{A}_{a b} \cdot \nabla w_{a b}+\frac{1}{\gamma_{a}} \mathbf{A}_{a} \cdot \nabla \gamma_{a}
\end{aligned}
$$

Thus the operators (17) verify the skew-adjonction property and variational calculus still holds, thus proving the consistency between the momentum equation and the continuity equation. The final term of $\operatorname{Grad}_{a}^{K}\left\{A_{b}\right\}$ was used by Kulasegaram et al. as a boundary force. In the following, we propose an enhanced form of the latter model, giving a more accurate representation of gradients on the wall.

\subsubsection{General shape of the boundary:}

For boundaries of arbitrary shape, their shape, $\partial \Omega$ of the domain $\Omega$, is approximated with straight segments in $2 D$ denoted by the subscript $(.)_{s}$ which have 

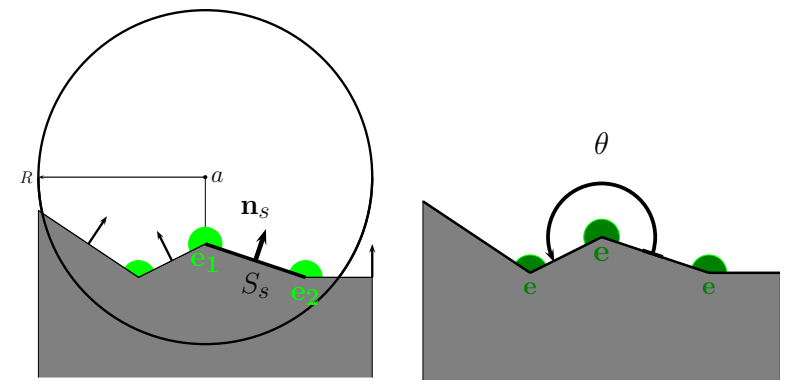

(a) Boundary with edge semi (b) Sketch of the volume of an particles $e$ (in green) and with edge particle. segments $s$ which have a surface $S_{s}$ and an inward normal $\mathbf{n}_{s}$.

Figure 2: Boundary property definitions.

a normal $\mathbf{n}_{s}$ and a surface area $S_{s}$ (see Figure 2(a)). The set containing all the segments is denoted by $\mathcal{S}$. Each segment is defined by two edge points denoted by the subscript (.) $)_{e}$ and (.) $)_{e 2}$ which have an initial volume $V_{e}$ given by $V_{e}=\frac{m_{e}}{\rho_{0}}$ where $\rho_{0}$ is a reference density. The initial volume of edge particles is a fraction of the initial volume of fluid particles $V_{f}$. For instance, for a plane $V_{e}=\frac{1}{2} V_{f}$. More generally, for an edge particle on a wedge with an angle $\theta$ displayed on the Figure 2(b), $V_{e}=\frac{\theta}{2 \pi} V_{f}$.

The set containing all the edge particles is denoted by $\mathcal{E}$. These edge particles (also called semi particles in this article) are of particular interest for recording the pressure field at the solid boundary (and hence for fluid and structure coupling for example). They are also useful to improve accuracy of the continuity equation, as they mimic a wet wall. It is important to notice that they are taken into account in the continuity equation and in the momentum equation (i.e. $\mathcal{E} \subset \mathcal{F}$ ), even if they are Eulerian particles, that is to say they are fixed if the wall is motionless and does not depend on the momentum equation.

Next we define the contribution of the segment $s$ in the value of $\nabla \gamma_{a}$ to be:

$$
\nabla \gamma_{a s} \equiv\left(\int_{\mathbf{r}_{e 1}}^{\mathbf{r}_{e 2}} w(r) \mathrm{d} l\right) \mathbf{n}_{s}
$$

and then $\nabla \gamma_{a}$ can be decomposed in:

$$
\nabla \gamma_{a}=\sum_{s \in \mathcal{S}} \nabla \gamma_{a s}
$$

The description of the boundary geometry can be extended to $3 D$ by substituting the segments by triangles. In this case this volume $V_{e}$ of edge particles would use the solid angle of the wedge.

\subsubsection{Wall corrected gradients:}

The main disadvantage of previous techniques to evaluate $\nabla \gamma_{a}$ is that the gradient operator defined by (17) is not accurate near a boundary or the free-surface. Indeed, we can see that gradients of constants are non zero: if we simulate uniform overpressure in a periodic pipe without any body force, the gradient (17) 
of the pressure is not zero everywhere and particles rearrange themselves (see Figure 3(a)). To correct that, we can go back to the continuous interpolation of an arbitrary integrable function $f$ at a point $\mathbf{r}$ :

$$
\langle f\rangle(\mathbf{r})=\frac{1}{\gamma(\mathbf{r})} \int_{\Omega \cap \Omega_{r}} f\left(\mathbf{r}^{\prime}\right) w(\tilde{r}) \mathrm{d} V^{\prime}
$$

where $\tilde{r} \equiv\left|\mathbf{r}-\mathbf{r}^{\prime}\right|$ and $\Omega_{r}$ is the kernel support centred in $\mathbf{r}$. By interpolating the gradient of the function $f$ in the same way, it becomes:

$$
\begin{aligned}
\langle\boldsymbol{\nabla} f\rangle(\mathbf{r})= & -\frac{1}{\gamma(\mathbf{r})} \int_{\Omega \cap \Omega_{r}} f\left(\mathbf{r}^{\prime}\right) \boldsymbol{\nabla} w(\tilde{r}) \mathrm{d} V^{\prime} \\
& -\frac{1}{\gamma(\mathbf{r})} \int_{\partial \Omega \cap \Omega_{r}} f\left(\mathbf{r}^{\prime}\right) w(\tilde{r}) \mathbf{n} \mathrm{d} S^{\prime}
\end{aligned}
$$

where the right-hand-side is obtained by an integration by parts and $\mathbf{n}$ is the inward normal of the domain at the position $\mathbf{r}^{\prime}$. Hence, we can see that the boundary conditions appear naturally through the second integral of (21).

Moreover, if we consider that the gradient defined by (7) is a discrete approximation of the continuous gradient $\boldsymbol{\nabla} f \equiv \rho \boldsymbol{\nabla} \frac{f}{\rho}+\frac{f}{\rho} \boldsymbol{\nabla} \rho$ (to obtain a symmetric formulation), we obtain (see also [6]):

$$
\begin{aligned}
\langle\boldsymbol{\nabla} f\rangle(\mathbf{r}) \simeq & \left\langle\rho(\mathbf{r}) \boldsymbol{\nabla} \frac{f}{\rho}+\frac{f}{\rho}(\mathbf{r}) \boldsymbol{\nabla} \rho\right\rangle(\mathbf{r}) \\
= & -\frac{1}{\gamma(\mathbf{r})} \int_{\Omega \cap \Omega_{r}}\left[\frac{f}{\rho}\left(\mathbf{r}^{\prime}\right) \rho(\mathbf{r})+\frac{f}{\rho}(\mathbf{r}) \rho\left(\mathbf{r}^{\prime}\right)\right] \boldsymbol{\nabla} w(\tilde{r}) \mathrm{d} V^{\prime} \\
& -\frac{1}{\gamma(\mathbf{r})} \int_{\partial \Omega \cap \Omega_{r}}\left[\frac{f}{\rho}\left(\mathbf{r}^{\prime}\right) \rho(\mathbf{r})+\frac{f}{\rho}(\mathbf{r}) \rho\left(\mathbf{r}^{\prime}\right)\right] w(\tilde{r}) \mathbf{n} \mathrm{d} S^{\prime}
\end{aligned}
$$

Once again the boundary conditions appear naturally, and we can now set the following discrete operator gradient for an arbitrary field $\left\{A_{b}\right\}$ as:

$$
\widetilde{\operatorname{Grad}}_{a}\left\{A_{b}\right\} \equiv \frac{\rho_{a}}{\gamma_{a}} \sum_{b \in \mathcal{F}} m_{b}\left(\frac{A_{a}}{\rho_{a}^{2}}+\frac{A_{b}}{\rho_{b}^{2}}\right) \nabla w_{a b}-\frac{\rho_{a}}{\gamma_{a}} \sum_{s \in \mathcal{S}}\left(\frac{A_{a}}{\rho_{a}^{2}}+\frac{A_{s}}{\rho_{s}^{2}}\right) \rho_{s} \nabla \gamma_{a s}
$$

The method to compute $\nabla \gamma_{a s}$ will be discussed in $\S 3.5$ whereas the computation of $\rho_{s}$ and $A_{s}$ will be investigated in the paragraph on dynamic boundary conditions 3.2.1. Notice that the discrete gradient (23) differs from Kulasegaram et al.'s defined by (17) only next to a boundary, which means that conservation properties are still valid "far from" the walls.

Let us now correct using the same idea the $S P H$ gradient of a vectorial field which appears in the velocity gradient (12) for instance. The latter quantity plays a key role in the $k-\epsilon$ turbulence model as it is responsible for the production of kinetic energy. Given the fact that in a channel flow the strain rate is the largest in the vicinity of the boundary, it is important to be accurate in this area. This formula needs to be corrected with respect to the boundaries, given that it tends to underestimate the strain rate next to a wall. To correct that we propose in a similar way as (23):

$$
\widetilde{\operatorname{Grad}}_{a}\left\{\mathbf{u}_{b}\right\}=-\frac{1}{\gamma_{a} \rho_{a}} \sum_{b \in \mathcal{F}} m_{b} \mathbf{u}_{a b} \otimes \nabla w_{a b}+\frac{1}{\gamma_{a} \rho_{a}} \sum_{s \in \mathcal{S}} \rho_{s} \mathbf{u}_{a s} \otimes \nabla \gamma_{a s}
$$




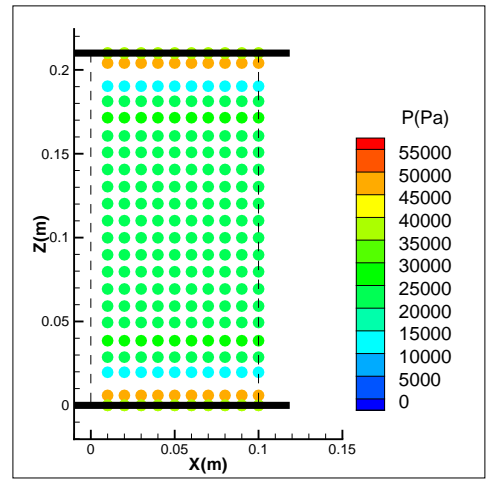

(a) Kulasegaram et al.'s gradient operator (17) is unable to maintain uniform pressure field. Particles move to an non-physical equilibrium.

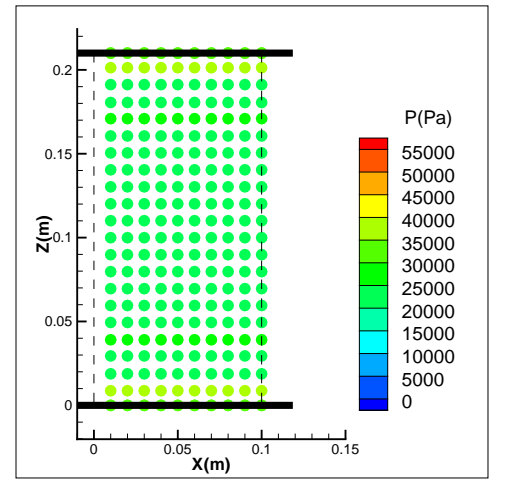

(b) The present gradient operator (23) is able to almost maintain uniform pressure field as an equilibrium.

Figure 3: Comparison of gradient operators (17) and (23) in an over-pressurized periodic pipe.

Hence, with the corrected components the strain rate $S$ is computed according to the definition given by (24).

\subsubsection{Laplacian:}

The Laplacian (7) resulting from the Morris model (5) can be seen as a discretisation of the continuous interpolation $\langle\nabla \cdot \mu \nabla f\rangle(\mathbf{r})$ (where $f$ is the continuous scalar field and $\mu$ the continuous viscosity field):

$$
\begin{aligned}
\langle\Delta(\mu, f)\rangle(\mathbf{r}) \equiv\langle\boldsymbol{\nabla} \cdot \mu \boldsymbol{\nabla} f\rangle(\mathbf{r})= & \left\langle\boldsymbol{\nabla}_{r^{\prime}} \cdot\left[(\mu \boldsymbol{\nabla} f)(\mathbf{r})+(\mu \boldsymbol{\nabla} f)\left(\mathbf{r}^{\prime}\right)\right]\right\rangle(\mathbf{r}) \\
= & -\frac{1}{\gamma(\mathbf{r})} \int_{\Omega \cap \Omega_{r}}\left[(\mu \boldsymbol{\nabla} f)(\mathbf{r})+(\mu \boldsymbol{\nabla} f)\left(\mathbf{r}^{\prime}\right)\right] \boldsymbol{\nabla} w\left(\mathbf{r}-\mathbf{r}^{\prime}\right) \mathrm{d} V^{\prime} \\
& -\frac{1}{\gamma(\mathbf{r})} \int_{\partial \Omega \cap \Omega_{r}}\left[(\mu \boldsymbol{\nabla} f)(\mathbf{r})+(\mu \boldsymbol{\nabla} f)\left(\mathbf{r}^{\prime}\right)\right] . \mathbf{n} w\left(\mathbf{r}-\mathbf{r}^{\prime}\right) \mathrm{d} S^{\prime}
\end{aligned}
$$

combined with the finite difference approximation:

$$
\boldsymbol{\nabla} f(\mathbf{r}) .\left(\mathbf{r}-\mathbf{r}^{\prime}\right) \simeq\left(f(\mathbf{r})-f\left(\mathbf{r}^{\prime}\right)\right) \simeq-\nabla f\left(\mathbf{r}^{\prime}\right) .\left(\mathbf{r}^{\prime}-\mathbf{r}\right)
$$

As in $\S 3.1$, the boundary terms appear naturally in the second line of (25) from an integration by parts. Again, $\mathbf{n}$ is the inward normal of the domain at the position $\mathbf{r}^{\prime}$. Wall contributions vanish when the position $\mathbf{r}$ is far from the boundary since the kernel $w$ has a compact support. Thus, the proposed wall-corrected Laplacian operator is:

$\frac{1}{\rho_{a}} \widetilde{\operatorname{Lap}}_{a}\left(\left\{B_{b}\right\},\left\{A_{b}\right\}\right)=\frac{1}{\gamma_{a}} \sum_{b \in \mathcal{F}} m_{b} \frac{B_{a}+B_{b}}{\rho_{a} \rho_{b}} \frac{A_{a b}}{r_{a b}^{2}} \mathbf{r}_{a b} \cdot \nabla w_{a b}-\frac{1}{\gamma_{a} \rho_{a}} \sum_{s \in \mathcal{S}}\left(B_{s} \nabla A_{s}+B_{a} \boldsymbol{\nabla} A_{a}\right) \cdot \boldsymbol{\nabla} \gamma_{a s}$

The same calculus still holds with an arbitrary vector field $\left\{\mathbf{A}_{b}\right\}$ and is used to correct the diffusion term (e.g. (5)) in the Navier-Stokes equation. 


\subsection{Wall corrected operators in the discrete Navier-Stokes equations}

\subsubsection{Dynamic boundary conditions on the pressure:}

The gradient operator (23) applied to the pressure field gives:

$$
\widetilde{\operatorname{Grad}}_{a}\left\{p_{b}\right\} \equiv \frac{\rho_{a}}{\gamma_{a}} \sum_{b \in \mathcal{F}} m_{b}\left(\frac{p_{a}}{\rho_{a}^{2}}+\frac{p_{b}}{\rho_{b}^{2}}\right) \nabla w_{a b}-\frac{\rho_{a}}{\gamma_{a}} \sum_{s \in \mathcal{S}}\left(\frac{p_{a}}{\rho_{a}^{2}}+\frac{p_{s}}{\rho_{s}^{2}}\right) \rho_{s} \nabla \gamma_{a s}
$$

Hence, we are left to compute the pressure $p_{s}$ and the density $\rho_{s}$ on the wall nodes defined in Figure 2(a). A robust way but first order dynamic condition is $\frac{\partial \rho}{\partial n}=0$. This condition is consistent with the renormalization done in equation (13). In the presence of gravity and motion the analogous condition on the pressure field is written as:

$$
\frac{\partial}{\partial n}\left(\frac{p^{\star}}{\rho}+\frac{u^{2}}{2}\right)=0
$$

where $p^{\star} \equiv p-\rho$ g.r and $u$ is the magnitude of the velocity.

To compute the pressure and the density at the wall an $S P H$ interpolation can be used for the edge particles in $\mathcal{E}$, the choice made is to average in space to give a wall value:

$$
\begin{aligned}
\rho_{e} & =\frac{1}{\alpha_{e}} \sum_{b \in \mathcal{F} \backslash \mathcal{E}} V_{b} \rho_{b} w_{b e} \\
\frac{p_{e}}{\rho_{e}} & =\frac{1}{\alpha_{e}} \sum_{b \in \mathcal{F} \backslash \mathcal{E}} V_{b}\left(\frac{p_{b}}{\rho_{b}}-\mathbf{g} \cdot \mathbf{r}_{b e}+\frac{u_{b}^{2}-u_{e}^{2}}{2}\right) w_{b e}
\end{aligned}
$$

where the set $\mathcal{F} \backslash \mathcal{E}$ denotes all fluid particles $\mathcal{F}$, excluding particles in $\mathcal{E}$ and where $\alpha_{e}$ is defined by:

$$
\alpha_{e} \equiv \sum_{b \in \mathcal{F} \backslash \mathcal{E}} V_{b} w_{b e}
$$

A description of the Shepard filter $\alpha_{e}$ is given in $\S 3.4 .1$ (see Equation 47)).

Here, it is important to note that the interpolation done is based on fluid particles in $\mathcal{F}$ which do not belong to the set of edge particles $\mathcal{E}$. For simplicity, we remain in $2 D$ and define the density and the pressure at the wall elements $s$ to be:

$$
\begin{aligned}
\rho_{s} & =\frac{\rho_{e 1}+\rho_{e 2}}{2} \\
\frac{p_{s}}{\rho_{s}} & =\frac{p_{e 1} / \rho_{e 1}+p_{e 2} / \rho_{e 2}}{2}
\end{aligned}
$$

where the wall nodes (or edge particles) $e 1$ and $e 2$ are defined in $\S 3.1 .2$. This strategy to evaluate quantities at the wall will be reused to compute the wall shear stress in $\S 3.2 .2$ and also the wall value of a scalar transported by the flow in Appendix A. There is no theoretical restriction to the spacing of the wall nodes. 


\subsubsection{Wall shear stress:}

The wall-corrected formula (27) applied to the field u gives:

$\frac{1}{\rho_{a}} \widetilde{\mathbf{L a p}}_{a}\left(\left\{\mu_{b}\right\},\left\{\mathbf{u}_{b}\right\}\right)=\frac{1}{\gamma_{a}} \sum_{b \in \mathcal{F}} m_{b} \frac{\mu_{a}+\mu_{b}}{\rho_{a} \rho_{b}} \frac{\mathbf{u}_{a b}}{r_{a b}^{2}} \mathbf{r}_{a b} . \nabla w_{a b}-\frac{1}{\gamma_{a} \rho_{a}} \sum_{s \in \mathcal{S}}\left|\boldsymbol{\nabla} \gamma_{a s}\right|\left(\mu_{a} \boldsymbol{\nabla} \mathbf{u}_{a}+\mu_{s} \boldsymbol{\nabla} \mathbf{u}_{s}\right) \cdot \mathbf{n}_{s}$

and should be used in place of $\operatorname{Lap}_{a}$ in (8-9).

The boundary terms are then treated using the friction velocity $\mathbf{u}_{\tau}$; defined by:

$$
\left.\mu \frac{\partial \mathbf{u}}{\partial \mathbf{n}}\right|_{\text {wall }} \equiv \rho u_{\tau} \mathbf{u}_{\tau}
$$

which represents the shear stress at the wall. In the present article, by convention, $\mathbf{u}_{\tau}$ is chosen to have the same direction as the local velocity field. This then replaces $(\mu \boldsymbol{\nabla u})_{s} \cdot \mathbf{n}_{s}$ in the boundary term of (33) as in classical finite element or finite volume Navier-Stokes codes:

$$
(\mu \nabla \mathbf{u})_{a} \cdot \mathbf{n}_{s} \simeq \rho u_{\tau s} \mathbf{u}_{\tau s}
$$

Computation of the friction velocity in a laminar case: The friction velocity $\mathbf{u}_{\tau}$ is a quantity defined at the boundary. To compute it in a Computational Fluid Dynamic ( $C F D$ ) code, instead of using its definition, we usually take the advantage of knowing the physical behaviour of the velocity field in the vicinity of the boundary. For example, in a laminar test case, the velocity profile is expected to be linear close to the wall and then the following relationship between distance to the wall $z$ and velocity along the wall $\mathbf{u}$ holds:

$$
u_{\tau} \mathbf{u}_{\tau}=\lim _{z \rightarrow 0} \frac{\nu \mathbf{u}}{z}
$$

The main advantage is that we do not need to estimate the derivative of the velocity field next to the wall, where it is difficult to compute. Another advantage is that we can extend the definition of the friction velocity in the area where particles interfere with the boundary, that is, when the kernel support intersects the walls. Hence, we define:

$$
u_{\tau a} \mathbf{u}_{\tau a}=\frac{\nu \mathbf{u}_{a}}{z_{a}}
$$

where $z_{a}$ is the distance to the wall for a particle $a$.

Eventually, to evaluate $\rho_{s} u_{\tau s} \mathbf{u}_{\tau s}$ we use again the continuity of stresses (35) to establish:

$$
\rho_{e} u_{\tau e} \mathbf{u}_{\tau e}=\frac{1}{\alpha_{e}} \sum_{b \in \mathcal{F} \backslash \mathcal{E}} V_{b} \rho_{b} u_{\tau b} \mathbf{u}_{\tau b} w_{b e}
$$

and $r h o_{s} u_{\tau s} \mathbf{u}_{\tau s}$ is the average value between the edge particles $e 1$ and $e 2$ defined in $\S 3.1 .2$. These formulae are similar to (32).

The same wall treatment is extended to a transport equation of a scalar such as $k$ or $\epsilon$ in the $k-\epsilon$ turbulence model in the Appendix A. 


\subsection{Conservation issues: time integration for the continu- ity equation}

The original time integration scheme used in previous work [13], [8] was a simple first-order symplectic scheme where an implicit velocity value was used in the updated values of the position and density:

$$
\left\{\begin{array}{l}
\mathbf{u}_{a}^{n+1}=\mathbf{u}_{a}^{n}-\frac{\delta t}{\rho_{a}^{n}} \operatorname{Grad}_{a}^{n}\left\{p_{b}^{n}\right\}+\mathbf{g} \\
\mathbf{r}_{a}^{n+1}=\mathbf{r}_{a}^{n}+\delta t \mathbf{u}_{a}^{n+1} \\
\rho_{a}^{n+1}=\rho_{a}^{n}+\delta t \sum_{b \in \mathcal{F}} m_{b} \nabla^{n} w_{a b} \cdot \mathbf{u}_{a b}^{n+1}
\end{array}\right.
$$

where the superscript $(.)^{n}$ refers to the time step $n$ and to the time $t=\sum_{i=1}^{n} \delta t$.

In this semi-implict scheme the velocities are explicit, whereas the positions are implicit. In the continuity equation, positions are explicit whereas the velocities are implicit; for this reason we do not write the r.h.s. of Equation (39) as $\rho_{a} \operatorname{Div}\left\{\mathbf{u}_{b}\right\}$.

\subsection{Improving the time integration of the continuity equa- tion}

To adapt the previous time integration scheme to the method of Kulasegaram et al. and the present modified one, the following scheme is possible:

$$
\left\{\begin{array}{l}
\mathbf{u}_{a}^{n+1}=\mathbf{u}_{a}^{n}-\frac{\delta t}{\rho_{a}^{n}}{\widetilde{\operatorname{Grad}_{a}^{n}}}_{\mathbf{r}_{a}^{n+1}}\left\{p_{b}^{n}\right\}+\mathbf{g} \\
\rho_{a}^{n+1}=\mathbf{r}_{a}^{n}+\delta t \mathbf{u}_{a}^{n+1} \\
\rho_{a}^{n}+\frac{\delta t}{\gamma_{a}^{n}}\left[\sum_{b \in \mathcal{F}} m_{b} \boldsymbol{\nabla}^{n} w_{a b} \cdot \mathbf{u}_{a b}^{n+1}-\rho_{a}^{n} \nabla^{n} \gamma_{a} \cdot \mathbf{u}_{a}^{n+1}\right]
\end{array}\right.
$$

where the operator $\widehat{\operatorname{Grad}}_{a}$ is either (17) or (23). Experience of the authors has shown this approach seems to give satisfactory results for a dam break case in a basin where the solid walls are kept impermeable with a relatively small time step. However, when running long-time simulations in a channel with a relatively large time step, particles near the wall move vertically downwards slowly and eventually pass through the boundary as we see in Figure 4(a) (a relatively big time step is set by decreasing the numerical speed of sound $c_{0}$ ) .

The problem is caused by the continuity equation: when particles near the boundary are oscillating, ie moving back and forth, their densities decrease, and then the pressure, related to the density by the equation of state, becomes insufficient to create a repulsive force to balance the other forces.

The origin of this phenomenon is the term $\frac{\delta t}{\gamma_{a}^{n}} \rho_{a}^{n} \nabla^{n} \gamma_{a} \cdot \mathbf{u}_{a}^{n+1}$ in the time discretized continuity equation (40). Indeed, if we consider a single particle moving towards the wall between the times $t_{n}$ and $t_{n+1}$ (from the distance $z_{n}$ to $z_{n+1}$ ), the exact variation of the density is not reproduced by the discrete 


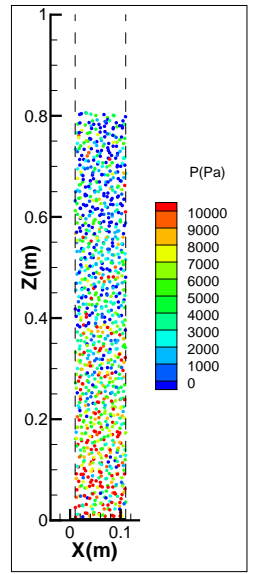

(a) Previous time scheme (40) with $c_{0}=20 m . s^{-1}$.

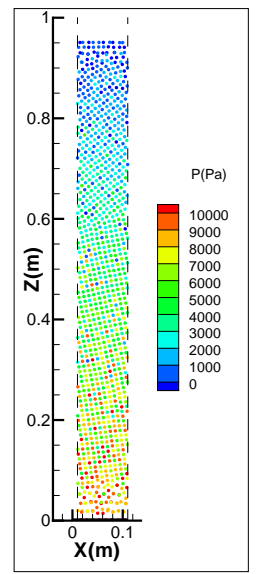

(b) Previous time scheme (40) with $c_{0}=100 m . s^{-1}$.

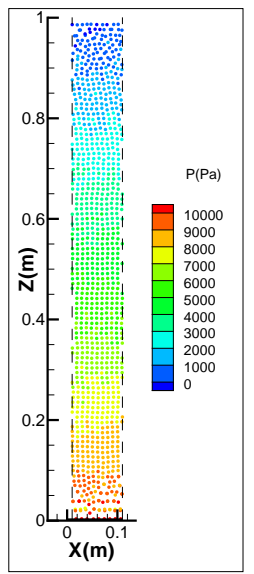

(c) New time scheme (42) with $c_{0}=20 m . s^{-1}$.

Figure 4: Comparison of the pressure field and the water depth in a periodic open channel flowing from left to right for two different time schemes after the same physical time.

form [15]. This systematic error of the time discretization of the continuity equation is undesirable.

Many other integration time schemes can be considered, such as a leap-frog time scheme, which would reduce the errors in the integration of the continuity equation. The choice made in the present work is to consider an approach that gives the density explicitly as a function of the particles' positions. This is a more robust approach, especially if there is a wall repulsive force function of the pressure field (and hence of the density field in compressible flows).

\subsubsection{A completely position-dependent way to compute the density:}

If we return to the main idea of correcting the incomplete kernel support, we can see that the corrected continuity equation comes from:

$$
\begin{aligned}
\frac{\mathrm{d}\left(\gamma_{a} \rho_{a}\right)}{\mathrm{d} t} & =\frac{\mathrm{d}}{\mathrm{d} t}\left(\sum_{b \in \mathcal{F}} m_{b} w_{a b}\right) \\
\gamma_{a} \frac{\mathrm{d} \rho_{a}}{\mathrm{~d} t}+\rho_{a} \frac{\mathrm{d} \gamma_{a}}{\mathrm{~d} t} & =\sum_{b \in \mathcal{F}} m_{b} \nabla w_{a b} \cdot \mathbf{u}_{a b}
\end{aligned}
$$

In a similar way, Vila [9] stated that the continuity equation (1) is strictly equivalent to $\frac{\mathrm{d} \rho_{a}}{\mathrm{~d} t}=\frac{\mathrm{d}}{\mathrm{d} t}\left(\sum_{b \in \mathcal{F}} m_{b} w_{a b}\right)$ if time is considered a continuous variable. Equation (41) gives us a way to integrate exactly in time the quantity $\gamma_{a} \rho_{a}$ if the particles' positions move from $\left\{\mathbf{r}_{b}^{n}\right\}$ to $\left\{\mathbf{r}_{b}^{n+1}\right\}$. This prevents systematic time integration errors, and makes $\rho_{a}^{n}$ depend only on the positions of particles 
at the same time (this property, together with simplectic time-stepping, ensures the conservation of a given energy, see [16] for details).

This leads to the following time integration scheme:

$$
\left\{\begin{aligned}
\mathbf{u}_{a}^{n+1} & \left.=\mathbf{u}_{a}^{n}-\frac{\delta t}{\rho_{a}^{n}}{\widetilde{\operatorname{Grad}_{a}^{n}}}_{a}^{n}\right\}+\mathbf{g} \\
\mathbf{r}_{a}^{n+1} & =\mathbf{r}_{a}^{n}+\delta t \mathbf{u}_{a}^{n+1} \\
\left(\gamma_{a} \rho_{a}\right)^{n+1} & =\left(\gamma_{a} \rho_{a}\right)^{n}+\sum_{b \in \mathcal{F}} m_{b}\left(w_{a b}^{n+1}-w_{a b}^{n}\right)
\end{aligned}\right.
$$

Figure 4 shows the result obtained by reducing the time-step (and hence the systematic error in the density equation) in the time scheme (40) with the result we obtain with the time-scheme (42) with a larger time step ${ }^{3}$. By doing so, scheme of (40) is forced to be stable and to converge but it is clear that we have lost density during the stabilisation time: the water depth has decreased (see 4(b)) whereas it is not the case on the Figure 4(c) with the new scheme.

Initialization of the density field: The time-scheme (42) requires initial values for the density field. Many choices are possible. First of all a particle $a$ can have the reference density $\rho_{0}$ as initial value, i.e.

$$
\rho_{a}^{0}=\rho_{0}
$$

This was done previously with the continuity equation of (39). The advantage is that the continuity equation only measures the variation of density and not the initial disorder of the particles. However, the main drawback is an inhomogeneity between particles could lead to non-physical behaviour, such as particles originally at the free-surface creating a region of repulsion when surrounded by others later in the simulation. Hence, the initial density field, $\left\{\rho_{a}^{0}\right\}$, is initialized according to:

$$
\rho_{a}^{0}=\frac{1}{\gamma_{a}^{0}} \sum_{b \in \mathcal{F}^{0}} m_{b} w_{a b}^{0}
$$

This initialization has the advantage to maintain homogeneity between particles, but then requires a free-surface correction.

Free-surface correction: The $\gamma$ correction presented so far does not take into account any free-surface correction. The variable $\tilde{\rho}_{a} \equiv \sum_{b \in \mathcal{F}} m_{b} w_{a b}$ depends only on the particles positions which is useful for conservation properties. The problem is that $\tilde{\rho}_{a}$ measures two different quantities:

1. the divergence of the particles, which is a quantity of interest, and

2. the presence of voids within the kernel support of a particle.

\footnotetext{
${ }^{3}$ We have reduced the time step by setting the speed of sound at $100 \mathrm{~m} \cdot \mathrm{s}^{-1}$ instead of $20 m . s^{-1}$
} 
It is required that the flow is corrected with $\gamma_{a}$ next to a wall, but not next to the free-surface. To achieve this, we use the following Shepard filter defined by:

$$
\alpha(\mathbf{r})=\sum_{b \in \mathcal{F}} \frac{m_{b}}{\rho_{b}} w\left(\mathbf{r}-\mathbf{r}_{b}\right)
$$

so that for a fluid particle $a \in \mathcal{F} \backslash \mathcal{E}$ we have:

$$
\alpha_{a} \equiv \sum_{b \in \mathcal{F}} \frac{m_{b}}{\rho_{b}} w_{a b}
$$

For an edge particle $e \in \mathcal{E}$ and for the middle of a segment $s \in \mathcal{S}$ two different definitions of $\alpha$ are used:

$$
\alpha_{e} \equiv \sum_{b \in \mathcal{F} \backslash \mathcal{E}} \frac{m_{b}}{\rho_{b}} w_{e b}
$$

since $\alpha_{e}$ is used to evaluate quantities such as the density or the pressure at the wall, hence we have chosen not to take into account edge particles and have interpolation using only the fluid particles of the physical quantities (such as the density $\rho$ or the pressure $p$ ) at the walls (see $\S 3.2 .1$ ).

The aim is to apply the Shepard filter (46) on the density field at each time step but only next to the free-surface such that the density field is not corrected everywhere with $\alpha_{a}$. Thus, in the vicinity of a free-surface there is a continuous mix to correct the continuity equation :

$$
\rho_{a}\left[\beta \gamma_{a}+(1-\beta) \alpha_{a}\right]=\tilde{\rho}_{a}=\sum_{b \in \mathcal{F}} m_{b} w_{a b}
$$

where

$$
\beta=\exp \left[-K\left(\min \left\{\frac{\alpha_{a}}{\gamma_{a}} ; 1\right\}-1\right)^{2}\right]
$$

and $K$ is taken to be an arbitrary high value of $-\frac{\ln (0.05)}{0.01^{2}} \simeq 30000$, so that $\beta \leq 0.05$ when $\frac{\alpha_{a}}{\gamma_{a}} \leq 0.99$. Note $\beta$ is in fact a surface-marker: inside the fluid its value is almost one whereas it tends to zero as we approach the free-surface.

\subsection{Computation of the renormalization terms}

The formal definitions of the geometrical quantities $\gamma_{a}$ and $\nabla \gamma_{a}$ for a particle $a$ are:

$$
\begin{aligned}
\gamma_{a} & \equiv \int_{\Omega \cap \Omega_{a}} w\left(\mathbf{r}-\mathbf{r}_{a}\right) \mathrm{d} \mathbf{r} \\
\nabla \gamma_{a} & \equiv \int_{\Omega \cap \Omega_{a}} \nabla_{a} w\left(\mathbf{r}-\mathbf{r}_{a}\right) \mathrm{d} \mathbf{r}=\int_{\partial \Omega \cap \Omega_{a}} w\left(\mathbf{r}-\mathbf{r}_{a}\right) \mathbf{n} \mathrm{d} S
\end{aligned}
$$

We recall that $\nabla \gamma_{a}$ represents an approximation of the normal to the wall for a particle located at the position $\mathbf{r}_{a}$. with previous approaches using polynomial approximation [1, 6], an analytical solution [10] , and a discrete summation over boundary points $[17]$. 
These approaches all had advantages and disadvantages discussed earlier. Herein, computation of the renormalization term of the kernel support near a solid wall is obtained with a time integration scheme, thereby more easily accounting for any shape of boundaries presented in $\S 3.1 .2$.

\subsubsection{Analytical value of $\nabla \gamma_{a}$ :}

With the boundary of the domain composed of segments denoted with the subscript $(.)_{s}$, each segment has an inward normal $\mathbf{n}_{s}$, a beginning point $\mathbf{r}_{e 1}$ and an ending point $\mathbf{r}_{e 2}$ (see Figure 2(a)). Then we can compute the analytical value of the contribution $\nabla \gamma_{a s}$ (defined by 18):

$$
\boldsymbol{\nabla} \gamma_{a s} \equiv\left(\int_{\mathbf{r}_{e 1}}^{\mathbf{r}_{e 2}} w(r) \mathrm{d} l\right) \mathbf{n}_{s}
$$

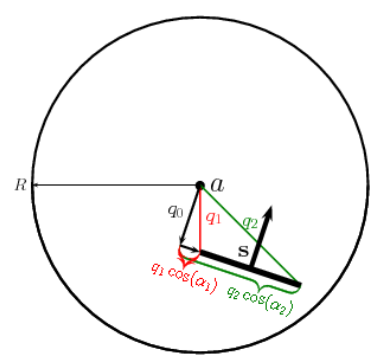

Figure 5: Schema showing the definitions of the geometrical parameters used to compute the analytical value of $\nabla \gamma_{a s}$ with (51).

which gives for the quintic Wendland kernel used in this work:

$$
\begin{aligned}
h \int_{\mathbf{r}_{e 1}}^{\mathbf{r}_{e 2}} w(r) \mathrm{d} l= & \frac{\left(q_{2} \cos \alpha_{2}\right)}{\pi} P_{q_{0}}\left(q_{2}\right)-\frac{\left(q_{1} \cos \alpha_{1}\right)}{\pi} P_{q_{0}}\left(q_{1}\right) \\
& +\frac{q_{0}^{4}}{\pi}\left(\frac{105}{64}+\frac{35}{512} q_{0}^{2}\right)\left[\begin{array}{l}
\operatorname{sign}\left(q_{2} \cos \alpha_{2}\right) \ln \left(\frac{q_{2}+\left|q_{2} \cos \alpha_{2}\right|}{\left|q_{0}\right|}\right) \\
-\operatorname{sign}\left(q_{1} \cos \alpha_{1}\right) \ln \left(\frac{q_{1}+\left|q_{1} \cos \alpha_{1}\right|}{\left|q_{0}\right|}\right)
\end{array}\right]
\end{aligned}
$$

where the polynomial function $P_{q_{0}}$ is defined by:

$$
\begin{aligned}
P_{q_{0}}(X)= & \frac{7}{192} X^{5}-\frac{21}{64} X^{4}+\frac{35}{32} X^{3}-\frac{35}{24} X^{2}+\frac{7}{4} \\
& +q_{0}^{2}\left(\frac{35}{768} X^{3}-\frac{7}{16} X^{2}+\frac{105}{64} X-\frac{35}{12}\right) \\
& +q_{0}^{4}\left(\frac{35}{512} X-\frac{7}{8}\right)
\end{aligned}
$$

where $q_{0} \equiv \frac{\left|\mathbf{r}_{a e i} . \mathbf{n}_{s}\right|}{h}, q_{i} \equiv \frac{\left|\mathbf{r}_{a e i}\right|}{h}$ and $q_{i} \cos \alpha_{i}(i \in\{1,2\})$ are displayed in Figure 5 . 
These analytical values enable estimates of the error due to the approximations. In Figure 6 we compare the analytical and approximate values of $\nabla \gamma_{a}$ against the distance to a plane wall. The discrete approximation used is given by:

$$
\nabla \gamma_{a s} \simeq w_{a s} S_{s} \mathbf{n}_{s}
$$

where it is assumed that the kernel is constant on a segment $s$.

The kernel used is the quintic kernel (see [18]) and the ratio $\frac{\delta r}{h}=2$ where $\delta r$ is the initial distance between two fluid particles and $h$ is the smoothing length. The absolute errors are defined as:

$$
\epsilon_{\nabla \gamma_{a}}=\frac{\left|\nabla \gamma_{a}^{\text {analytical }}-\nabla \gamma_{a}^{\text {discrete }}\right|}{\nabla \gamma_{a}^{\text {analytical }}}
$$

(We consider only the component of $\nabla \gamma_{a}$ orthogonal to the wall). In Figure 6, the error for the discretization of $\nabla \gamma_{a}$ is very good for such a poor discretization ratio $\frac{\delta r}{h}$ for a plan wall (less than $0.1 \%$ ) whereas the discretization has a systematic error for the approximation of the value of $\gamma_{a}$ on the order of $3 \%$.

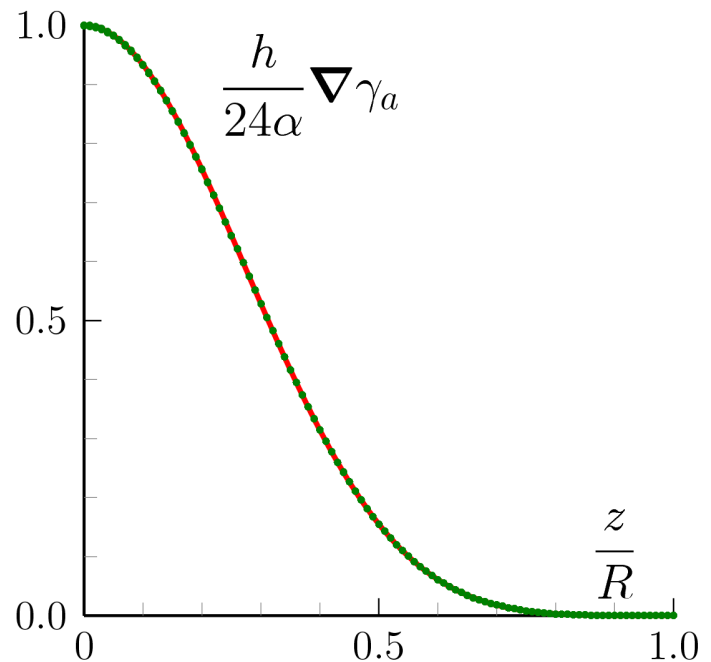

(a) Values of the $\nabla \gamma_{a}$ function against the distance of the wall.

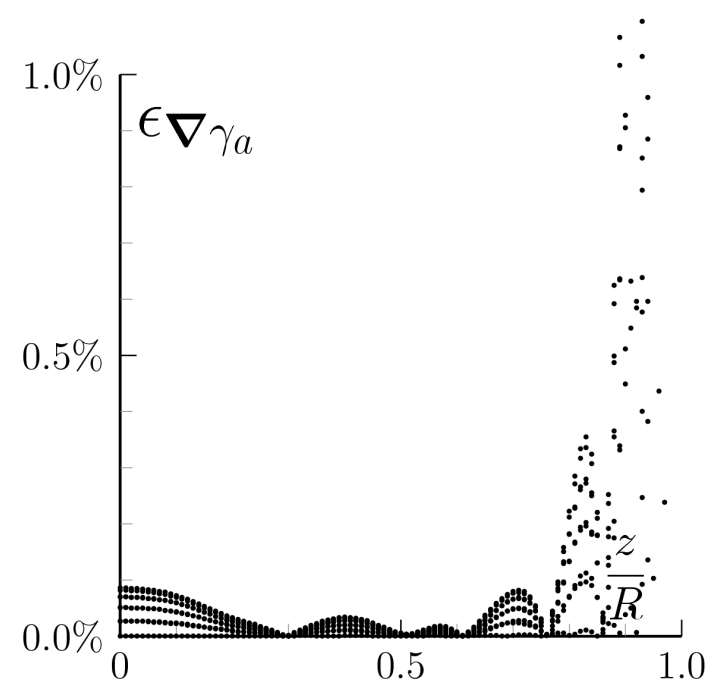

(b) Values of the error function $\epsilon \nabla \gamma_{a}$ against the distance of the wall.

Figure 6: Analytical in red and computed values in green of the functions against the distance to a plan wall.

If the discretization error of $\nabla \gamma_{a}$ in the presence of a complex boundary such as a wedge it was found that the error is larger. Moreover, the error is systematic, so that the magnitude of $\boldsymbol{\nabla} \gamma_{a}$ is always underestimated. Within a simulation, this leads to non-physical behaviour, i.e. particles slide towards along wedge boundary since the gravity is not balanced completely by the wall repulsive force proportional to $\boldsymbol{\nabla} \gamma_{a}$. 


\subsubsection{Governing equation for $\gamma_{a}$ :}

Herein, a method to compute $\gamma_{a}$ for a particle $a$ near a solid boundary is suggested without the need for fictitious particles and is therefore simpler than an analytic computation. The main idea of the present method is to use a governing equation of $\gamma_{a}$ :

$$
\left\{\begin{array}{rlr}
\frac{\mathrm{d} \gamma_{a}}{\mathrm{~d} t} & =\boldsymbol{\nabla} \gamma_{a} \cdot \mathbf{u}_{a} & \\
\gamma_{a} & =1 & \text { if } \partial \Omega \cap \Omega_{a}=\emptyset
\end{array}\right.
$$

where the definition of the gradient is combined with the fact that $\frac{\mathrm{d} \mathbf{r}_{a}}{\mathrm{~d} t}=\mathbf{u}_{a}$. Another way to consider this equation is to remark that (55) is equivalent to:

$$
\left\{\begin{aligned}
\frac{\partial \gamma_{a}}{\partial t} & =0 \\
\gamma_{a} & =1 \text { if } \partial \Omega \cap \Omega_{a}=\emptyset
\end{aligned}\right.
$$

This means that the $\gamma_{a}$ field does not depend on the time ${ }^{4}$, but only on position, and is therefore an Eulerian field. Thus, it gives us a means to compute $\gamma_{a}$ to be coherent with $\nabla \gamma_{a}$ which is easier to compute, since it can be expressed as a surface integral.

The relation (55) can be extended for moving boundaries. Recalling the boundary defined by Figure 2(a), if we have a moving deformable wall (in the sense that each segment or triangle composing the wall is moving with its velocity) the following formula is obtained:

$$
\left\{\begin{array}{rlrl}
\frac{\mathrm{d} \gamma_{a}}{\mathrm{~d} t} & =\sum_{s \in \mathcal{S}} \boldsymbol{\nabla} \gamma_{a s} \cdot \mathbf{u}_{a}^{\mathcal{R}_{s}} & \\
\gamma_{a} & =1 & & \text { if } \partial \Omega \cap \Omega_{a}=\emptyset
\end{array}\right.
$$

where $\mathbf{u}_{a}^{\mathcal{R}_{s}}$ is the velocity of the particle $a$ in a reference frame $\mathcal{R}_{s}$ where the segment $s$ is fixed. The field $\gamma_{a}$ is now computed by solving the above equation, based on the knowledge of $\nabla \gamma_{a s}$, computed from (51).

\subsubsection{Initialization of the $\gamma_{a}$ field:}

The initialization step of $\gamma_{a}^{0}$ is done by imaging a virtual transformation. For each particle initially next to a solid wall (a criterion is $\left|\nabla \gamma_{a}^{0}\right|>0$ ), we move it from its starting position $\mathbf{r}_{a}^{0}$ to an area where the function $\gamma(\mathbf{r}) \equiv 1$. For instance:

$$
\mathbf{r}_{a}=\mathbf{r}_{a}^{0}+l \frac{\boldsymbol{\nabla} \gamma_{a}^{0}}{\left|\nabla \gamma_{a}^{0}\right|}
$$

where the length $l$ is taken to be $2 R,(R$ is the radius of the compact kernel support).

A sketch of the proposed method is displayed in Figure 7. For example, the circled particle in Figure 7 is placed behind the dashed line where the value of

\footnotetext{
${ }^{4}$ if the wall does not move.
} 
$\gamma$ is 1 , and is moved back to its initial position along the path of the large black arrow updating the value of $\gamma_{a}$ with respect to the governing Equation (57).

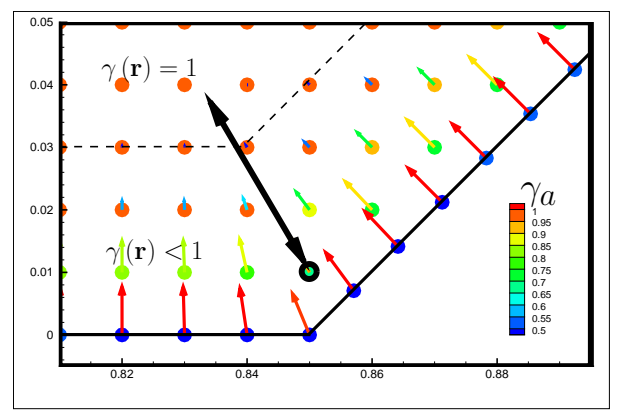

Figure 7: Sketch of the initialization of the $\gamma$ field next to a solid wall.

Note that the equation of $\gamma_{a}$ is integrated in time with a second-order time integration scheme to prevent systematic integration errors (see § 3.4), leading to:

$$
\left\{\gamma_{a}^{n+1}=\gamma_{a}^{n}+\frac{1}{2}\left(\nabla^{n} \gamma_{a}+\nabla^{n+1} \gamma_{a}\right) \cdot\left(\mathbf{r}_{a}^{n+1}-\mathbf{r}_{a}^{n}\right)\right.
$$

if the solid boundary is motionless. The general formula for a moving deformable wall is:

$$
\left\{\gamma_{a}^{n+1}=\gamma_{a}^{n}+\frac{\delta t}{2} \sum_{s \in \mathcal{S}}\left(\nabla^{n} \gamma_{a s}+\nabla^{n+1} \gamma_{a s}\right) \cdot\left(\mathbf{u}_{a}^{\mathcal{R}_{s}}\right)^{n+1}\right.
$$

A condition on the time step is required, to keep the integration of $\gamma_{a}$ stable:

$$
\delta t \leq C_{t, \gamma} \frac{1}{\max _{a \in \mathcal{F} ; s \in \mathcal{S}}\left\{\left|\nabla^{n} \gamma_{a s} \cdot\left(\mathbf{u}_{a}^{\mathcal{R}_{s}}\right)^{n}\right|\right\}}
$$

where $C_{t, \gamma}=0.005$, from numerical experience. This is a natural condition, in the sense that the time step decreases when particles posses a fast velocity when approaching a boundary such as splashing against a wall. The latter condition is considered in addition to the usual time-stepping ( $C F L)$ conditions in $S P H$ (see e.g. [7]).

\section{Numerical results}

\subsection{Laminar channel flow test case}

To test the friction terms and validate the wall shear stress formulation, laminar Poiseuille flow in a closed-channel with periodic open boundaries is simulated . The channel has a diameter of $1 \mathrm{~m}$ while the viscosity $\nu$ is set at $10^{-1} \mathrm{~m}^{2} \cdot \mathrm{s}^{-1}$ so that the Reynolds number is 10 . The viscous term is modelled with the wall-corrected model of Morris (33) combined with (37) to compute the friction velocity. Figure 8 shows that the horizontal velocity profile is in good agreement with the analytical solution (62) even in the vicinity of the wall, thus demonstrating that the wall shear stress correctly balances the body force. 

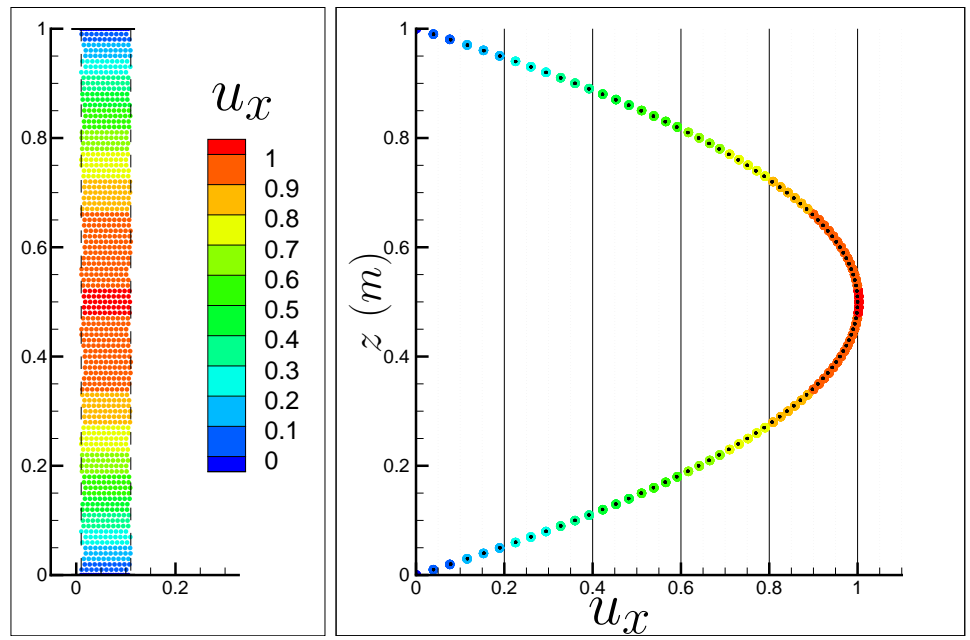

Figure 8: Poiseuille flow in a periodic pipe with a Reynolds number of 10. Coloured dots represent the velocity of particles at the steady state whereas the black dots • are the analytical profile (62).

Computation of the strain: The laminar case allows us to validate formula (24), since there is no influence of the strain rate on the flow itself because the viscosity is constant. Thus, such a test case allows us to compare the value of the computed strain rate to its analytical value. The analytical profile of velocity is:

$$
u_{x}(z)=4 R e \frac{\nu z}{D^{2}}\left(1-\frac{z}{D}\right)
$$

which leads to the following analytical value of $S$ :

$$
S(z)=4 R e \frac{\nu}{D^{2}}\left|2 \frac{z}{D}-1\right|
$$

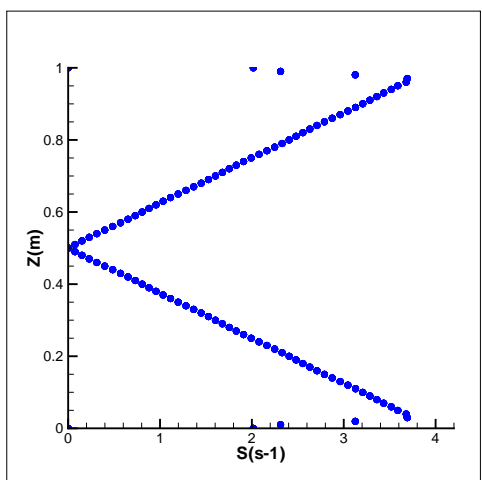

(a) Standard model (12)

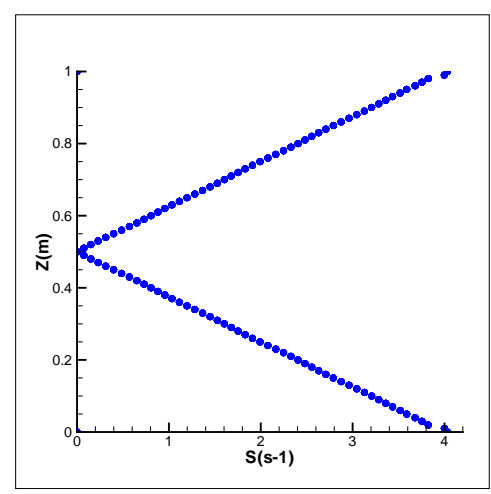

(b) Correction of Standard model (24)

Figure 9: Comparison of the strain rate for different models in a laminar channel flow.

We notice in Figure 9 that in this theoretical test case, the corrected method 
(24) gives a satisfactory reproduction of the shear stress next to the wall unlike the previous methods.

\subsection{Still water and dam break in a tank with a wedge}

In this section, the proposed scheme is tested on a more complex geometry which consists of a basin of approximately $2 m$ length and $1 m$ height with a wedge of $\frac{\pi}{2} \mathrm{rad}$ angle and $\frac{\sqrt{2}}{8} m$ of height in the bottom middle of the tank. This geometry was chosen specifically since the wedge features both a discontinuous point and a sloping profile which tests the correction of the kernel in the presence of gravity. A comparison is made between different models in a still water case and a dynamic case where the viscosity $\nu$ is set at $10^{-2} \mathrm{~m}^{2} \cdot \mathrm{s}^{-1}$.

\subsubsection{Still water case:}

Some treatments for solid boundaries suffer from an inability to reproduce correctly a still water case. Here we compare the results obtained when the basin is filled with $0.5 \mathrm{~m}$ of water for three cases: the Lennard-Jones repulsive forces (see [7]), the fictitious particles method (see [8]) and then new proposed method. As expected, the repulsive forces produce poor results (see the Figures 10(b)) in the sense that particles keep sliding along vertical wall. That is due to the fact that the missing area in the kernel support is not compensated, and thus the gravity is not balanced sufficiently. The plot of the pressure of particles against the depth is therefore noisy and badly reproduced next to the bottom. The fictitious particles method (see Figure 10(a)) gives better results, but the condition (29) is not ensured and so the pressure profile is still noisy. Moreover this approach is problematic to describe in complex geometries and requires additive particles to mimic the boundary, which increase the computation cost. The present method gives superior results: a linear pressure profile even near the bottom and a zero velocity field as shown in Figure 10(c).

\subsubsection{Dynamic case:}

A simulation of a dam break with the same geometry has been performed for the two boundary techniques previously described and the present one. The water is initially a column of $1 m$ height and $0.5 m$ width on the left-hand side of the basin. In the results shown in Figure 11, all approaches ensure impermeable boundaries, but both of repulsive forces method and fictitious particles method give a noisier pressure field. Furthermore, a simulation with finer resolution has been computed by doubling resolution and hence the number of particles. Snapshots of the pressure field at the same physical time are plotted on the Figure 11.

The comparison with a VOF simulation (done with Finite Volume opensource code OpenFoam) of the pressure on the left side of the wedge is perfomed and displayed on Figure 12. 

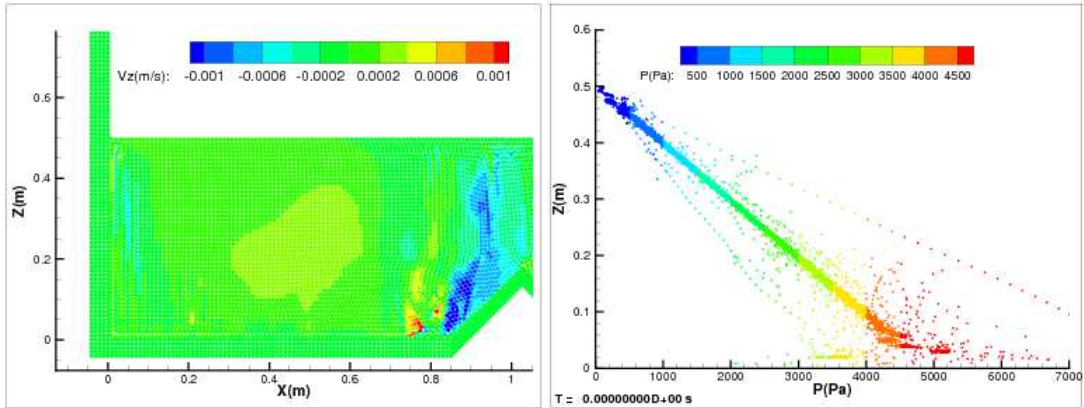

(a) Fictitious particles.

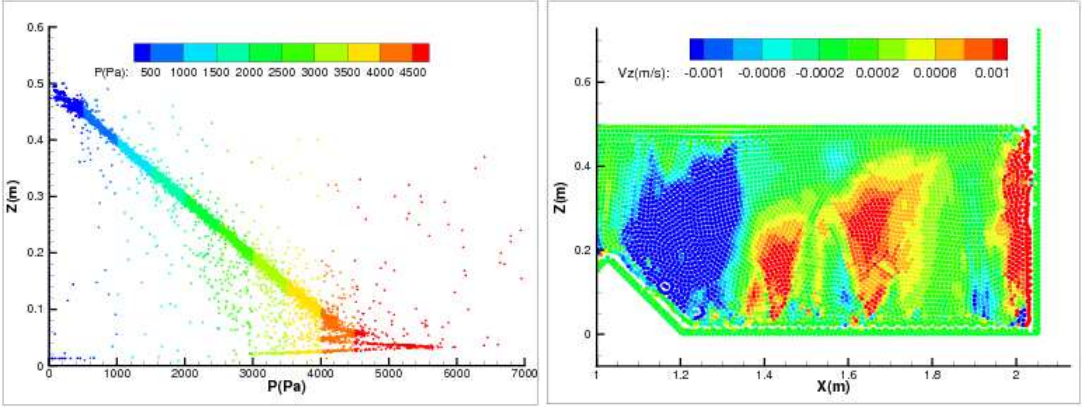

(b) Lennard-Jones type repulsive force.
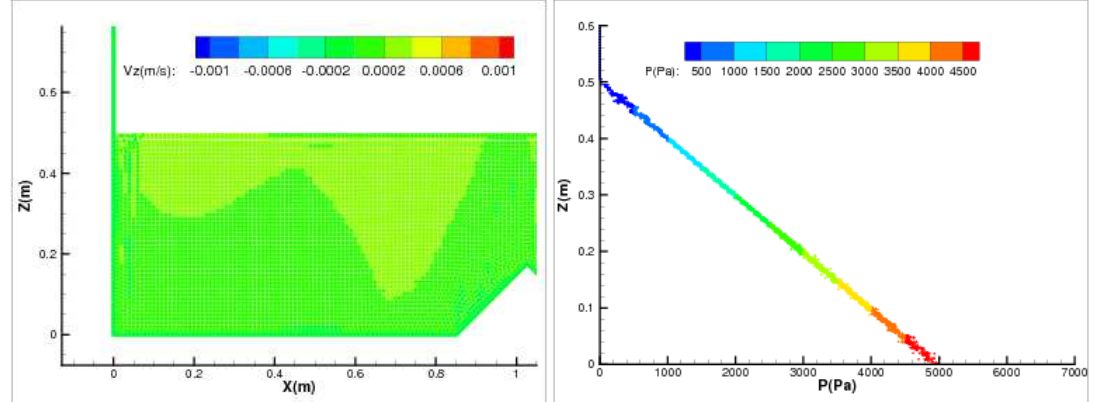

(c) Present method.

Figure 10: Comparison of the vertical velocity for still water in a tank with a wedge for different boundary conditions after $20 \mathrm{~s}$.

\subsection{Comparison of the $k-\epsilon$ model with $S P H$ and Finite Volumes: simulation of a fish pass}

Once a dam is built on a river, the continuity of the flow is disrupted and the migration of fish is interrupted. For species such as some salmon, the life cycle requires the fishes to migrate far upriver. To restore the migration process, fish passes are installed which consist of many repeating elements and can be considered as a periodic flow. The dimensioning of these components requires a detailed knowledge of the turbulent flow within each section since the size of the large eddies affects the ability of the fish to swim upstream. 


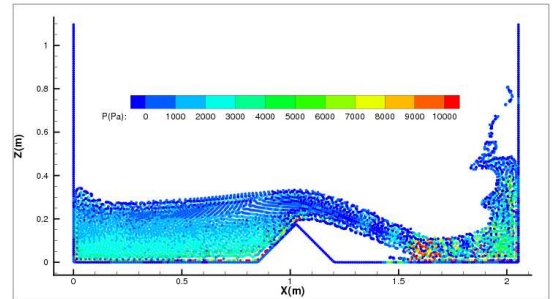

(a) Lennard and Jones repulsive forces.

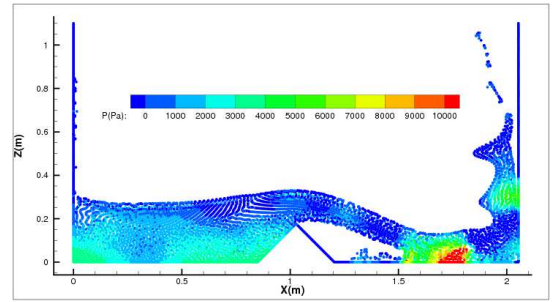

(c) Present method.

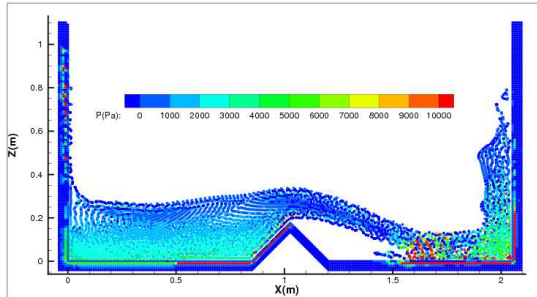

(b) Fictitious particles.

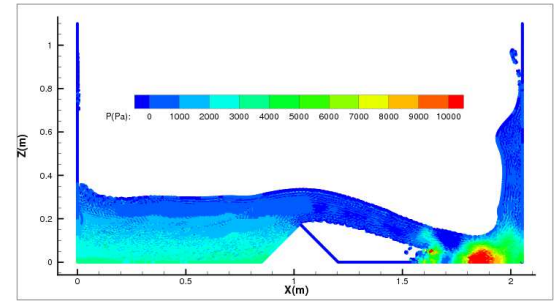

(d) Present method with a twice smaller space discretization.

Figure 11: Comparison of the pressure field for a dam break test case in a tank with a wedge for different boundary conditions.

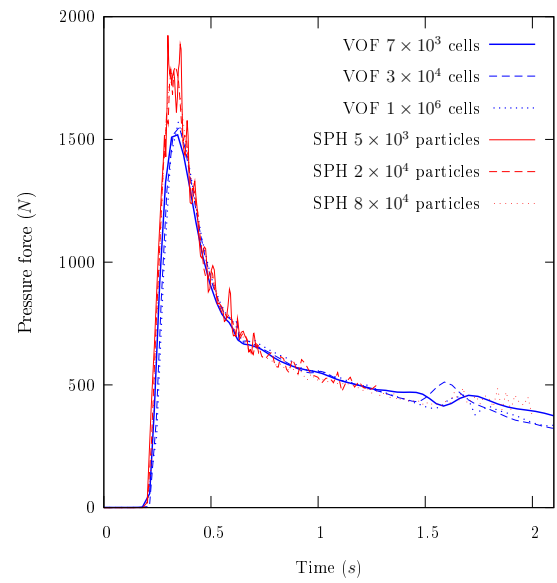

Figure 12: Comparison of the time evolution of the pressure along the left side of the wedge between the present $S P H$ formulation and the finite volume code OpenFoam at different spacial discretization.

Although the behaviour of the flow though a fish pass is a $3 D$ free-surface flow, we consider here $2 D$ simulations where the vertical variation are assumed to be negligible. We repeat here the simulations presented in Violeau et al. [19]. The geometry of the $x$-periodic simulation is presented in Figure 13(a). The results obtained by $S P H$ are compared to simulations done with Code_Saturne, a widely validated Finite Volume code developed by EDF RESD (see [20]). The aim of such a comparison is to evaluate the performance of $S P H$ for a turbulent simulation. The computation is compared effectively solving the same equations 
(the Reynolds-averaged Navier Stokes with the $k-\epsilon$ model) with the same pressure gradient responsible driving the flow $\left(\frac{\Delta p}{\rho \Delta x}=1.885 \mathrm{~m} . \mathrm{s}^{-2}\right)$ but with two different approaches (Lagrangian and Eulerian) with two different discretization approaches ( $S P H$ and Finite Volume).

The velocity profiles at locations $P_{1}, P_{2}$ and $P_{3}$ defined in Figure 13(a) are plotted in Figure 14. The results show that the mesh-based code gives profiles close to the ones obtained with the present $S P H$ scheme. It should be noted that with the standard SPH method [19], the predicted velocity and eddy viscosity distributions did not fit the Finite Volume ones. With the present model, one can see that the agreement is very satisfactory.

\section{Conclusion}

The present article has presented a new approach to deal with solid boundary condition which is both simple and robust. The simplicity lies in the manner we compute the Eulerian renormalization term $\gamma_{a}$ with a integration in time which only requires the computation of its gradient $\nabla \gamma_{a}$. The robustness is due to the integration in time of the continuity equation which makes the density field depend only on the particles' positions. This allows a long time simulation with a relatively higher time step and is a major advantage for conservation properties.

The definition of new boundary corrected gradient and Laplacian operators gives us the opportunity to fix boundary conditions and fluxes on the pressure field, the wall shear stress and even the scalar fields such as $k$ and $\epsilon$ in a model of turbulence.

However, numerous issues still require investigation and development, namely:

1. Validate the present formulation on different test case such as a periodic turbulent bump.

2. Adapt the wall renormalization to $3 D$; the main challenge is to find an analytical formula for the computation of the contribution of a surface element $s$ over the value of $\nabla \gamma_{a}$ of a fluid particle $a$ (which is denoted by $\left.\nabla \gamma_{a s}\right)$, or a way to compute accurately an approximated value of it.

3. Study the theoretical conservation (or non-conservation) of momentum and angular momentum, and especially in periodic cases.

4. Combine the present approach with non-periodic entrance conditions.

\section{A Turbulent wall conditions in $S P H$}

\section{A.1 Computation of the friction velocity in a turbulent case}

We can apply the same wall correction of the diffusion term of the momentum equation as the laminar one, noticing that $\left(\mu+\mu_{T}\right) \mathbf{S} . \mathbf{n} \simeq \rho u_{\tau} \mathbf{u}_{\tau}$ in the vicinity 


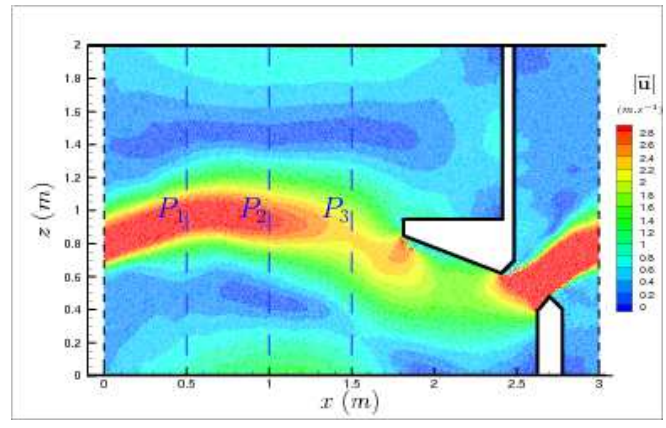

(a) Magnitude of the velocity field with SPARTACUS-2D.

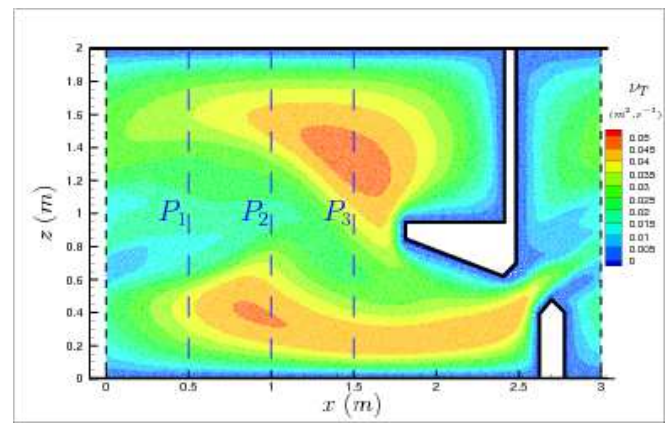

(c) Turbulent viscosity $\nu_{T}$ field with SPARTACUS$2 D$.

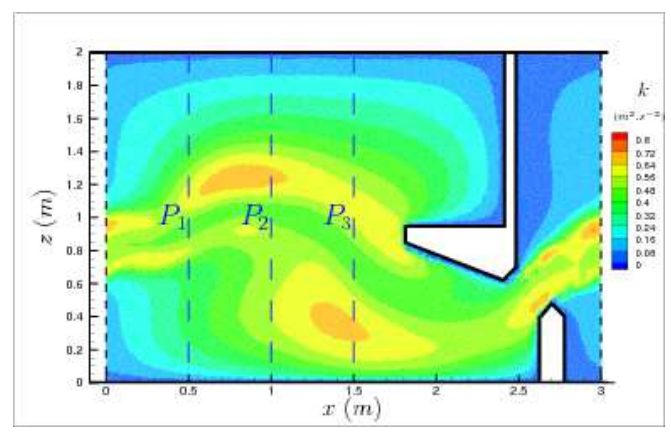

(e) Kinetic energy $k$ field with SPARTACUS-2D.

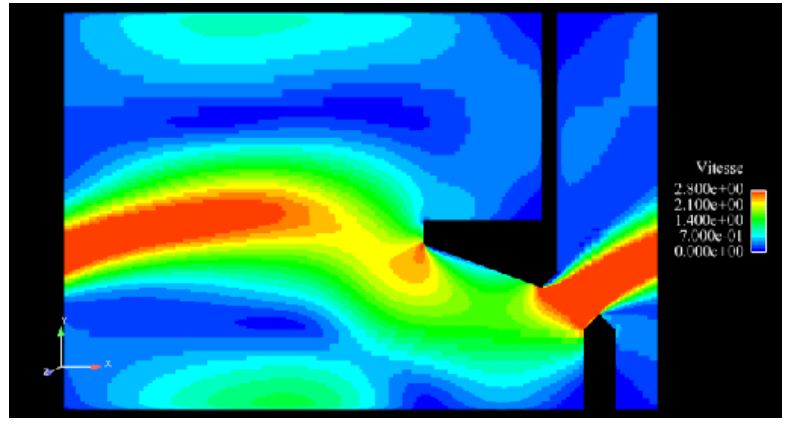

(b) Turbulent viscosity $\nu_{T}$ field with Code_Saturne.

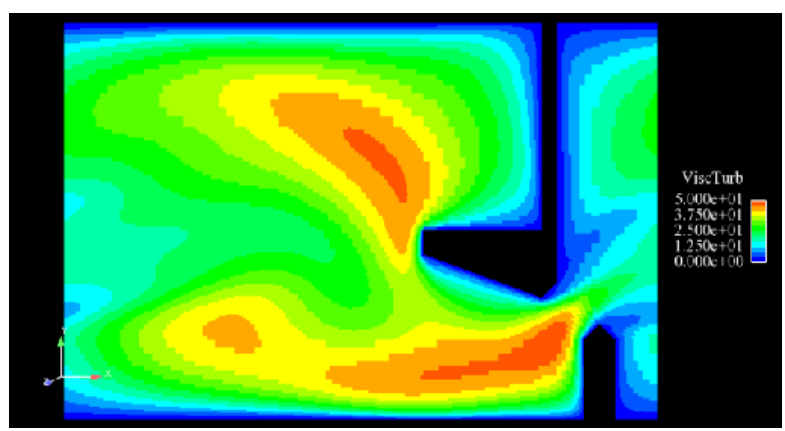

(d) Turbulent viscosity $\nu_{T}$ field with Code_Saturne.

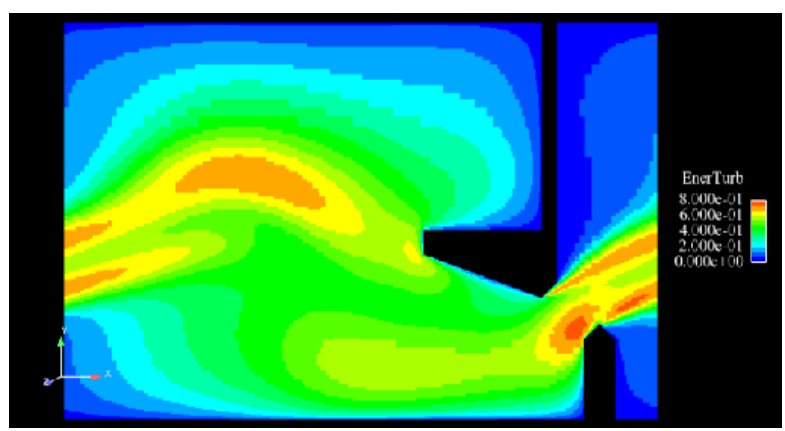

(f) Kinetic energy $k$ field with Code_Saturne.

Figure 13: Comparison of the $k-\epsilon$ model (9) with Lagrangian $S P H$ approach and an Eulerian Finite Volume method in a schematic fish pass.

of a wall ${ }^{5}$. Unlike the Equation (37) which links friction velocity to the mean velocity, in the turbulent case, we need another wall function. The viscosity is not constant anymore, and is supposed to be linear in the vicinity of a wall ${ }^{6}$. Then it can be shown that the velocity profile in that area is a logarithmic shape: this zone is called the $\log$ layer. If we consider the particle $a$ to be in

\footnotetext{
${ }^{5}$ Formally, at a wall $k=0$ and then $\nu_{T}=0$ so that we recover the laminar case. However, the viscous sub-layer where the laminar viscosity is more important than the turbulent one is usually, for environmental flows, very thin so that we do not enforce $k$ to be zero at a wall.

${ }^{6}$ In a channel flow, this assumption is well verified in $10 \%$ of the channel depth.
} 


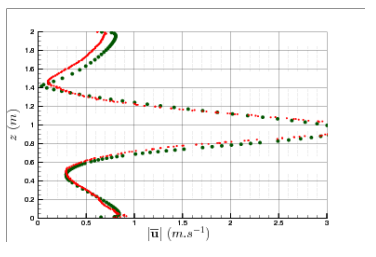

(a) Profiles in $P_{1}$.

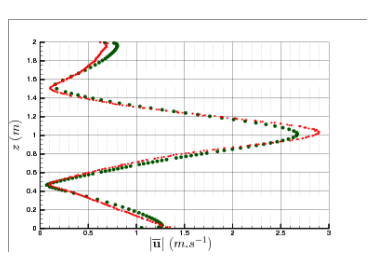

(b) Profiles in $P_{2}$.

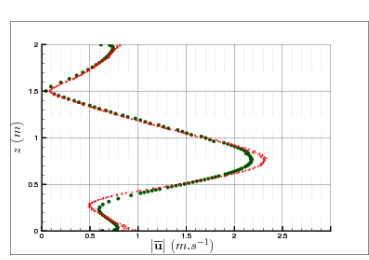

(c) Profiles in $P_{3}$.

Figure 14: Profiles of the velocity magnitude in three different plans in the fish pass.The $k-\epsilon$ model (9) in red and the $k-\epsilon$ model with Code_Saturne in green.

the $\log$ layer of a smooth wall, $u_{\tau}$ can be obtained from the following wall law, with an iterative algorithm:

$$
\frac{\left|\mathbf{u}_{a}\right|}{u_{\tau a}}=\frac{1}{\kappa} \ln \left(\frac{z_{a} u_{\tau a}}{\nu}\right)+5.2
$$

To be valid, Equation (64) must verify that the non-dimensional distance to the wall $\frac{z_{a} u_{\tau a}}{\nu}$ is greater than 11 . We could also use log laws for rough walls, or use laws which hold both in the laminar and the log layer such as Reichards' law, but then the $k-\epsilon$ model (9) must also be modified for low Reynolds effects. For more information see [21].

\section{A.2 Velocity at the wall}

We observe in the formula (24) that the velocity at the wall $\mathbf{u}_{s}$ is considered in the boundary term. Formally, the no-slip condition would impose that the velocity at the wall is the velocity of the wall itself (i.e. 0 for a motionless wall). This is imposed for a laminar flow. In the turbulent case, it is preferable to not do so: the slope of the velocity profile is much larger at the wall than in the $\log$ layer where fluid particles are assumed to be. Thus if we want to evaluate accurately $S_{a}$ next to the wall, we need to interpolate the velocity at the wall. To do so, we let the velocity $\mathbf{u}_{e}$ of edge particles balance the viscous and friction terms:

$$
\frac{\mathrm{d} \mathbf{u}_{e}}{\mathrm{~d} t}=\underbrace{\frac{1}{\gamma_{e}} \sum_{b \in \mathcal{F}} m_{b} \frac{\mu_{T e}+\mu_{T b}}{\rho_{e} \rho_{b}} \frac{\mathbf{u}_{e b}}{r_{e b}^{2}} \mathbf{r}_{e b} . \nabla w_{e b}}_{\text {viscous term }}-\underbrace{\frac{2 u_{\tau e} \mathbf{u}_{\tau e}}{\gamma_{e}} \sum_{s \in \mathcal{S}}\left|\nabla \gamma_{e s}\right|}_{\text {friction term }}
$$

We notice here that Equation (65) is the momentum equation applied to an edge particle with neither gravity nor pressure gradient. And we define $\mathbf{u}_{s}$ to be the average value between wall particles $e 1$ and $e 2$ defined in $\S 3.1 .2$. This tactic to allow a kind of "slip" velocity at the wall in a high Reynolds number simulation is also used in many $C F D$ codes such as TELEMAC-3D in Finite Elements (see Hervouet [22]). Eventually, we have to bear in mind that the edge particles in $\mathcal{E}$ are in fact Eulerian points and do not move with the velocity $\mathbf{u}_{e}$ but with the wall velocity. That is to say we only use the velocity $\mathbf{u}_{e}$ to update viscous forces of fluid particles interacting with the wall, and to compute the strain rate $S$. 


\section{A.3 Flux conditions on the kinetic energy}

The Laplacian operator (27) applied to the turbulent kinetic energy reduces to:

$$
\frac{1}{\rho_{a}} \widetilde{\operatorname{Lap}_{a}}\left(\left\{\mu_{b}+\frac{\mu_{T b}}{\sigma_{k}}\right\},\left\{k_{b}\right\}\right)=\frac{1}{\gamma_{a}} \sum_{b \in \mathcal{F}} m_{b} \frac{2 \mu+\mu_{T a} / \sigma_{k}+\mu_{T b} / \sigma_{k}}{\rho_{a} \rho_{b}} \frac{k_{a b}}{r_{a b}^{2}} \mathbf{r}_{a b} \cdot \nabla w_{a b}
$$

because it is assumed that there is no flux of $k$ from the boundary, i.e. $\frac{\partial k}{\partial n}=$ 0 at the wall. Equation (66) should be used in place of $\operatorname{Lap}_{a}$ in (9). The physical meaning is that the turbulent kinetic energy is only created by the mean flow. Moreover, instead of specifying boundary conditions at the wall, the "wall function" approach describes the balance of terms in the vicinity of the wall where it is assumed that $P=\epsilon$ : this implies that the condition $\frac{\partial k}{\partial n}=0$ is valid not only at the wall, but in the whole vicinity of the solid boundary.

If we assume the flow to be highly turbulent, that is to say, with a thin viscous sub-layer, we can use high Reynolds number laws for $k-\epsilon$. In fact we do not solve the $k-\epsilon$ model up to the wall, where $k$ is theoretically expected to be 0 , but up to a small distance $\delta$ from the wall, where the turbulence is fully established $\left(\nu_{T} \gg \nu\right)$. The main advantage of the present Lagrangian approach compared to an Eulerian one is that all the Lagrangian fluid particles in $\mathcal{F} \backslash \mathcal{E}$ will be at least at a distance of the order of $\delta r$ from any wall. It is one of the main advantages compared to the mesh-based methods where the boundary elements are supposed to be at a virtual distance to the actual wall, here we only focus on particles which are effectively at a non-zero distance from the walls.

To estimate $k$ at the wall we can evaluate as we did for the density, the pressure or the wall shear stress:

$$
k_{e}=\frac{1}{\alpha_{e}} \sum_{b \in \mathcal{F} \backslash \mathcal{E}} V_{b} k_{b} w_{b e}
$$

We notice that this approximation is consistent with the assumption $\frac{\partial k}{\partial n}=0$. Finally:

$$
k_{s}=\frac{k_{e 1}+k_{e 2}}{2}
$$

\section{A.4 Flux conditions on the dissipation of kinetic energy}

The Laplacian operator (27) applied to $\epsilon$ requires the value for $\partial \epsilon / \partial n$. Once again, if we assume that the flow is highly turbulent, then every single particle in the area of influence of a wall (i.e. $\exists s \in \mathcal{S} /\left|\nabla \gamma_{a s}\right|>0$ ) is in the log layer where $^{7}$ :

$$
\begin{aligned}
k & \simeq \frac{u^{\star 2}}{\sqrt{C_{\mu}}} \\
\epsilon & =\frac{u^{\star 3}}{\kappa z} \\
\nu_{T} & =\kappa u^{\star} z
\end{aligned}
$$

\footnotetext{
${ }^{7}$ All the Equations (69) can be derived from the equilibrium $P=\epsilon$.
} 
where $z$ is the distance to the wall (if a particle $a$ is interacting with a surface $s$, we state $\left.z=\max \left(\mathbf{r}_{a s} . \mathbf{n}_{s} ; \delta r\right)\right)$, where $\delta r$ is the particle averaged initial spacing, $\kappa$ is the Von Karman constant with the value of 0.41 , and $u^{\star}$ is a friction velocity measuring the turbulence:

$$
u_{s}^{\star}=\frac{\sqrt{k_{s}}}{C_{\mu}^{\frac{1}{4}}}
$$

Then, from Equation (69) we can deduce a value for the flux of $\epsilon$ :

$$
\frac{\nu_{T a}}{\sigma_{\epsilon}} \frac{\partial \epsilon_{a}}{\partial n_{s}}=-\frac{2 u_{s}^{\star 4}}{\sigma_{\epsilon} \kappa \delta r_{a s}}
$$

The factor 2 is provided by a first-order approximation (i.e the flux is evaluate at the distance $\frac{z}{2}$ ). This accuracy is needed especially here because $\epsilon$ is supposed to vary as $\frac{1}{z}$ where $z$ is the distance to the wall.

Thus the Laplacian (27) becomes:

$$
\begin{aligned}
\frac{1}{\rho_{a}} \widetilde{\operatorname{Lap}_{a}}\left(\left\{\mu_{b}+\frac{\mu_{T b}}{\sigma_{\epsilon}}\right\},\left\{\epsilon_{b}\right\}\right)= & \frac{1}{\gamma_{a}} \sum_{b \in \mathcal{F}} m_{b} \frac{2 \mu+\mu_{T a} / \sigma_{\epsilon}+\mu_{T b} / \sigma_{\epsilon}}{\rho_{a} \rho_{b}} \frac{\epsilon_{a b}}{r_{a b}^{2}} \mathbf{r}_{a b} . \nabla w_{a b} \\
& +\frac{4}{\gamma_{a} \rho_{a}} \sum_{s \in \mathcal{S}}\left|\nabla \gamma_{a s}\right| \rho_{s} \frac{u_{s}^{\star 4}}{\sigma_{\epsilon} \kappa \delta r_{a s}}
\end{aligned}
$$

which is used in place of $\operatorname{Lap}_{a}$ in (9).

\section{References}

[1] Kulasegaram S, Bonet J, Lewis RW, Profit M. A variational formulation based contact algorithm for rigid boundaries in two-dimensional sph applications 2004; .

[2] Oger G, Doring M, Alessandrini B, Ferrant P. An improved sph method: Towards higher order convergence. Journal of Computational Physics 2007; 225(2):1472 - 1492 .

[3] Di Monaco A, Manenti S, Gallati M, Sibilla S, Agate G, Guandalini R, Maffio A. A semi-analytic approach for sph modelling of solid boundaries. $4^{\text {th }}$ SPHERIC workshop, Nantes, France, 2009; $165-172$.

[4] Monaghan J, Kajtar J. Sph particle boundary forces for arbitrary boundaries. Computer Physics Communications 2009; 180(10):1811 - 1820.

[5] Marongiu JC, Leboeuf F, Caro J. Low mach number numerical schemes for the sph-ale method. application in free surface flows in pelton turbines. $4^{\text {th }}$ SPHERIC workshop, Nantes, France, 2009; 323 - 330.

[6] De Leffe M, Le Touzé D, Alessandrini B. Normal flux method at the boundary for sph. $4^{\text {th }}$ SPHERIC workshop, Nantes, France, 2009; $149-156$.

[7] Monaghan JJ. Simulating free surface flows with SPH. Journal of Computational Physics 1994; 110:399-406. 
[8] Violeau D, Issa R. Numerical modelling of complex turbulent free-surface flows with the sph method: an overview. International Journal for $\mathrm{Nu}$ merical Methods in Fluids 2007; 53(2):277-304, doi:10.1002/fld.1292. URL http://dx.doi.org/10.1002/fld.1292.

[9] Vila JP. Weighted particle previous termmethodsnext term and smooth particle hydrodynamics. Math. Models Meth. Appl. Sci. 1999; 9(2):161209.

[10] Feldman J, Bonet J. Dynamic refinement and boundary contact forces in sph with applications in fluid flow problems. Int. Journal for Numerical Methods in Engineering 2007; 72(3):295 - 324. Meshfree Methods: Recent Advances and New Applications.

[11] Monaghan JJ. Smoothed particle hydrodynamics. Annual review of Astronomy and Astrophysics 1992; 30:543-574.

[12] Morris JP, Fox PJ, Zhu Y. Modeling low reynolds number incompressible flows using sph. Journal of Computational Physics 1997; 136:214-226.

[13] Violeau D. The Mechanics of weakly compressible flows and its application to the SPH method. to be published, 2010.

[14] Launder BE, Spalding DB. Mathematical models of turbulence. London: Academic Press 1972; .

[15] Ferrand M, Laurence D, Rogers B, Violeau D. Improved time scheme integration approach for dealing with semi analytical boundary conditions in spartacus2d. Proc. $V^{\text {th }}$ SPHERIC International Workshop, Manchester, UK, 2010.

[16] Goldstein H, Poole C, Safko J. Conservation of the Hamiltonian. Addison Wesley, San Francisco, 2002. $3^{\text {rd }}$ edition.

[17] Marongiu JC, Leboeuf F, Parkinson E. Numerical simulation of the flow in a Pelton turbine using the meshless method SPH and a new simple solid boundary treatment. 7th European Conference on Turbomachinery Fluid Dynamics and Thermodynamics, Athens, Greece, 2007.

[18] Wendland H. Piecewise polynomial, positive definite and compactly supported radial functions of minimal degree. Advances in Computational Mathematics December 1995; 4(1):389-396.

[19] Violeau D, Issa R, Benhamadouche S, Saleh K, Chorda J, Maubourguet MM. Modelling a fish passage with sph and eulerian codes: the influence of turbulent closure. Proc. III ${ }^{\text {rd }}$ SPHERIC International Workshop, Lausanne, Suisse, 2008; 85-91.

[20] Code_Saturne : a Finite Volume Code for the Computation of Turbulent Incompressible Flows - Industrial Applications, vol. 1. International Journal on Finite Volumes, 2004.

[21] Pope SB. Turbulent Flows. Cambridge University Press, 2000.

[22] Hervouet J. Hydrodynamics of Free Surface Flows: Modelling with the Finite Element Method. John Wiley, 2007. 